\title{
Low-frequency adsorbate vibrational relaxation and sliding friction
}

\author{
B. N. J. Persson \\ Abdus Salam International Center for Theoretical Physics, P.O. Box 586, I-34014 Trieste, Italy \\ and Institut für Festkörperforschung, Forschungszentrum Jülich, D-52425 Jülich, Germany \\ E. Tosatti \\ Abdus Salam International Center for Theoretical Physics, P.O. Box 586, I-34014 Trieste, Italy \\ and Scuola Internazionale Superiore di Studi Avanzanti, Via Beirut 2, I-34014, Trieste, Italy \\ D. Fuhrmann, G. Witte, and Ch. Wöll \\ Ruhr-Universität Bochum, Lehrstuhl für Physikalische Chemie I, Universitätsstraße 150, D-44801 Bochum, Germany
}

(Received 9 November 1998)

\begin{abstract}
We present a study of the damping (energy relaxation) of low-frequency adsorbate vibrational modes via one-phonon emission. The dependence of the damping rate on the size of the adsorbates, the adsorbate coverage, and the nature of the (nonphononic) adsorbate-adsorbate interactions has been studied. We show that different experimental probing techniques, e.g., inelastic helium scattering or time-resolved adsorbate response to substrate heating, probe different damping rates. We also show that the energy relaxation rate may be strongly affected by (pure) dephasing processes. Experimental data are presented and compared to the theoretical results. Finally, we show that the phononic friction vanishes for incommensurate layers sliding on solid surfaces and present a discussion about the nature of the surfaces used in recent measurement of the sliding friction on superconducting lead surfaces. [S0163-1829(99)07417-2]
\end{abstract}

\section{INTRODUCTION}

Physisorbed and weakly chemisorbed adsorbates on surfaces can perform low-frequency vibrational motion where the molecules vibrate parallel or perpendicular to the surface. These so-called frustrated translational modes are of fundamental importance for various dynamical processes, such as diffusion, desorption, reaction, and sliding friction. ${ }^{1,2}$ Thermal excitation of the low-frequency modes also influences the vibrational absorption spectra of high-frequency modes via anharmonic coupling, resulting in dephasing of the highfrequency modes. Thus, the $\mathrm{C}-\mathrm{O}$ stretch vibration of adsorbed $\mathrm{CO}$ is usually strongly affected by the anharmonic coupling to the parallel CO vibrations: thermal excitation of the latter modes results in both a line broadening and frequency shift of the $\mathrm{C}-\mathrm{O}$ stretch infrared-absorption profile. ${ }^{3}$

An adsorbate vibrational mode, with a resonance frequency $\omega_{0}$ below the maximum bulk phonon frequency $\omega_{c}$, forms a resonance in the bulk phonon continuum. In the absence of pure dephasing and inhomogeneous broadening, the width of such a resonance equals $1 / \tau$, where $\tau$ is the energy relaxation time or simply the "lifetime" of the mode. If $\omega_{0}$ is in the linear region of the dispersion relation of the bulk (and surface) phonon modes (which usually is the case if $\omega_{0}<0.3 \omega_{c}$ ), then the phonon contribution to the damping $\eta=1 / \tau$ can be accurately calculated using the elastic continuum model. On metals there will also be an electronic contribution to $\eta$ but this is often small compared with the phononic contribution. ${ }^{4}$ Exceptions to this general rule are observed when chemical interactions lead to a mixing of the substrate electronic states with the adsorbed molecule. ${ }^{5}$

The phonon contribution to the damping of low-frequency adsorbate modes has been studied within the elastic con- tinuum model by Persson and Ryberg. ${ }^{6}$ They focused on the dilute limit where the interference between the elastic waves emitted from the adsorbates can be neglected. The damping of vibrations in ordered adsorbate layers has been studied by Hall et al. ${ }^{7}$ and, more recently, by Bruch and Hansen ${ }^{8}$ and Lewis et al. ${ }^{9,10}$ The latter authors concluded that, in general, the dilute limit studied by Persson and Ryberg is valid only for adsorbate coverage below $1 \%$. However, we will show in this paper that this is not the case in many important applications, such as (a) the adsorbate-substrate energy transfer in response to substrate heating, (b) the dephasing of highfrequency modes, and (c) the energy transfer at an interface between a fluid and a solid, where the excitation and deexcitation of the adsorbate vibrational modes occur incoherently (in contrast to infrared spectroscopy where only the $\mathbf{q}=\mathbf{0}$ phonon mode is excited). In all these important applications the phononic damping calculated in the dilute limit should be quite accurate also at high adsorbate coverage. This conclusion differs from that of Lewis et al., who applied their high coverage damping formula (valid for the $\mathbf{q}=\mathbf{0}$ mode) to study the adsorbate-substrate energy transfer for $\mathrm{CO}$ on $\mathrm{Cu}(111)$, in response to ultrafast laser heating of the substrate. ${ }^{12}$

In this paper we generalize the results presented above to adsorbates of arbitrary size (but treated as rigid objects), which is relevant for, e.g., "large", hydrocarbons. We also discuss the influence of adsorbate-adsorbate interactions of a nonphononic nature, i.e., not mediated by the substrate phonons, on the damping rate. We show how the damping depends on the nature of the vibrational excitations, e.g., how it differs for coherent (say, the $\mathbf{q}=\mathbf{0}$ mode) and incoherent excitations in ordered adsorbate films. Experimental data are presented and compared to the theoretical results. 


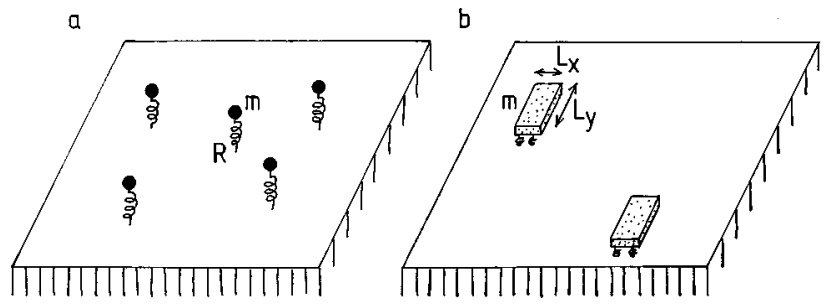

FIG. 1. (a) Atomic adsorbates (mass $m$ ) bound at points $\{\mathbf{R}\}$ on a semi-infinite elastic solid. (b) The same as (a) but for "large", molecular adsorbates, treated as rectangular "blocks.",

Finally, we consider the (adsorbate $\rightarrow$ substrate) energy transfer during sliding of incommensurate adsorbate layers on solids, which is relevant for the interpretation of the sliding friction measurements of Dayo, Alnasrallah, and Krim, ${ }^{13}$ and present a discussion about the nature of the surfaces used in recent measurement of the sliding friction on superconducting lead surfaces.

\section{GENERAL THEORY}

The theory outlined below is an extension of the results presented in Refs. 6-11, and we refer the readers to these references for more details. Let us consider a layer of adsorbates (mass $m$ ) bound by harmonic springs (with a spring constant $m \omega_{0}^{2}$ ) to a set of points $\{\mathbf{R}\}$ on the surface of a semi-infinite elastic solid [see Fig. 1(a)]. We consider first oscillations of the particles parallel to the surface. Let us introduce a coordinate system with the $x y$ plane in the surface with the $z$ axis pointing into the solid and the $x$ axis along the direction of oscillations. If $s_{\mathbf{R}}$ denotes the $x$ component of the displacement vector of the particle at $\mathbf{x}=\mathbf{R}$, then

$$
m \ddot{s}_{\mathbf{R}}=-m \omega_{0}^{2}\left[s_{\mathbf{R}}-u_{1}(\mathbf{R}, 0)\right]+F_{\mathbf{R}},
$$

where $u_{1}(\mathbf{R}, 0)$ is the $x$ component of the displacement vector of the substrate at the point $(\mathbf{R}, z=0)$. Both $s_{\mathbf{R}}$ and $u_{1}$ depend, of course, on time $t$, but the time dependence, which we always assume to be of the form $\exp (-i \omega t)$, will not be shown explicitly in what follows. The driving force $F_{\mathbf{R}}$ in (1) may have different physical origins; e.g., in an inelastic helium scattering experiment it is associated with the heliumadsorbate interaction, but the exact origin of this force is of no importance for the following consideration.

The oscillating adsorbates will give rise to a stress acting on the surface $(z=0)$ of the elastic solid, which in the simplest case is of the form

$$
\sigma_{13}=\sum_{\mathbf{R}} m \omega_{0}^{2}\left[u_{1}(\mathbf{R}, 0)-s_{\mathbf{R}}\right] \delta(\mathbf{x}-\mathbf{R}),
$$

where $\mathbf{x}=(x, y)$ is the two-dimensional position vector in the $z=0$ plane. We now generalize this result by assuming that the force on the substrate from an adsorbate is not localized to a single point $\mathbf{x}=\mathbf{R}$, but is distributed uniformly over a rectangular area of length $L_{x}$ in the $x$ direction and $L_{y}$ in the $y$ direction. This generalization is unimportant (and not meaningful) for small adsorbates such as $\mathrm{CO}$, but is important for large molecules, e.g., for "large" hydrocarbons. For perpendicular vibrations in "large" physisorbed adsorbates the assumption of a uniformly distributed adsorbate-substrate stress is likely to be a reasonable approximation. For parallel vibrations the situation is less clear, and it will depend on the particular system under study. Thus, we replace Eq. (2) with [see Fig. 1(b)]

$$
\sigma_{13}=\sum_{\mathbf{R}} m \omega_{0}^{2}\left[\bar{u}_{1}(\mathbf{R}, 0)-s_{\mathbf{R}}\right] \Pi(\mathbf{x}-\mathbf{R}),
$$

where $\Pi(\mathbf{x})=1 /\left(L_{x} L_{y}\right)$ if $-L_{x} / 2<x<L_{x} / 2$ and $-L_{y} / 2<y$ $<L_{y} / 2$, and zero otherwise, and where

$$
\bar{u}_{1}(\mathbf{R}, 0)=\int d^{2} x \Pi(\mathbf{x}-\mathbf{R}) u_{1}(\mathbf{x}, 0) .
$$

Note that

$$
\Pi(\mathbf{x})=\frac{1}{L_{x}} \int_{-L_{x} / 2}^{L_{x} / 2} d x^{\prime} \delta\left(x-x^{\prime}\right) \frac{1}{L_{y}} \int_{-L_{y} / 2}^{L_{y} / 2} d x^{\prime} \delta\left(y-y^{\prime}\right) .
$$

Using

$$
\delta\left(x-x^{\prime}\right)=\frac{1}{2 \pi} \int d k_{x} e^{i k_{x}\left(x-x^{\prime}\right)}
$$

and the corresponding expression for $\delta\left(y-y^{\prime}\right)$ gives

$$
\Pi(\mathbf{x})=\frac{1}{4 \pi^{2}} \int d^{2} k \frac{\sin \left(k_{x} L_{x} / 2\right)}{\left(k_{x} L_{x} / 2\right)} \frac{\sin \left(k_{y} L_{y} / 2\right)}{\left(k_{y} L_{y} / 2\right)} e^{i \mathbf{k} \cdot \mathbf{x}} .
$$

Substituting this result in Eq. (3) gives

$$
\sigma_{13}=\int d^{2} k f(\mathbf{k}) e^{i \mathbf{k} \cdot \mathbf{x}},
$$

where

$$
\begin{aligned}
f(\mathbf{k})= & \frac{m \omega_{0}^{2}}{4 \pi^{2}} \frac{\sin \left(k_{x} L_{x} / 2\right)}{\left(k_{x} L_{x} / 2\right)} \frac{\sin \left(k_{y} L_{y} / 2\right)}{\left(k_{y} L_{y} / 2\right)} \\
& \times \sum_{\mathbf{R}}\left[\bar{u}_{1}(\mathbf{R}, 0)-s_{\mathbf{R}}\right] e^{-i \mathbf{k} \cdot \mathbf{R}} .
\end{aligned}
$$

Using the theory of elasticity it is now straightforward to calculate the elastic displacement field $\mathbf{u}$. In what follows we only need the displacement field at the surface $z=0$. The displacement field $\mathbf{u}$ in the substrate satisfies

$$
-\omega^{2} \mathbf{u}-c_{L}^{2} \nabla \nabla \cdot \mathbf{u}+c_{T}^{2} \nabla \times(\nabla \times \mathbf{u})=0,
$$

where $c_{L}$ and $c_{T}$ are the longitudinal and transverse sound velocities, respectively. The general solutions to this equation can be written as a sum of plane waves:

$$
\mathbf{u}=\sum_{\mathbf{k}}\left(\mathbf{A}_{\mathbf{k}} e^{i\left(\mathbf{k} \cdot \mathbf{x}+p_{L} z-\omega t\right)}+\mathbf{B}_{\mathbf{k}} e^{i\left(\mathbf{k} \cdot \mathbf{x}+p_{T} z-\omega t\right)}\right),
$$

where

$$
p_{L}=\left(\frac{\omega^{2}}{c_{L}^{2}}-k^{2}\right)^{1 / 2} \text { and } p_{T}=\left(\frac{\omega^{2}}{c_{T}^{2}}-k^{2}\right)^{1 / 2}
$$

are the $z$ components of the wave vectors of the longitudinal and transverse waves, respectively. Note that $p_{L}$ and $p_{T}$ are imaginary for $k>\omega / c_{L}$ and $k>\omega / c_{T}$, respectively, and in these cases the plane waves become evanescent (they decay 
exponentially with increasing $z$ ) and no transfer of lattice energy (phonons) to the substrate is possible. The general solution (6) contains parameters which must be chosen so that the stress on the surface $z=0$ equals the driving stress exerted on the substrate by the oscillating adsorbates given by Eq. (4). This gives (see Ref. 6)

$$
u_{1}(\mathbf{x}, 0)=-\frac{i}{\rho c_{T}^{2}} \int d^{2} k f(\mathbf{k})\left(\frac{k_{y}^{2}}{k^{2} p_{T}}+\frac{\omega^{2} k_{x}^{2} p_{T}}{c_{T}^{2} k^{2} P(k)}\right) e^{i \mathbf{k} \cdot \mathbf{x}},
$$

with

$$
P=\left(2 k^{2}-\frac{\omega^{2}}{c_{T}^{2}}\right)^{2}+4 p_{T} p_{L} k^{2}
$$

From Eq. (8) we obtain

$$
\begin{aligned}
\bar{u}_{1}(\mathbf{R}, 0)= & -\frac{i}{\rho c_{T}^{2}} \int d^{2} k f(\mathbf{k})\left(\frac{k_{y}^{2}}{k^{2} p_{T}}+\frac{\omega^{2} k_{x}^{2} p_{T}}{c_{T}^{2} k^{2} P(k)}\right) \\
& \times \frac{\sin \left(k_{x} L_{x} / 2\right)}{\left(k_{x} L_{x} / 2\right)} \frac{\sin \left(k_{y} L_{y} / 2\right)}{\left(k_{y} L_{y} / 2\right)} e^{i \mathbf{k} \cdot \mathbf{R} .}
\end{aligned}
$$

Substituting Eq. (5) into Eq. ( $\left.8^{\prime}\right)$ gives

$$
\bar{u}_{1}(\mathbf{R}, 0)=\sum_{\mathbf{R}^{\prime}} \chi\left(\mathbf{R}-\mathbf{R}^{\prime}\right)\left[\bar{u}_{1}\left(\mathbf{R}^{\prime}, 0\right)-s_{\mathbf{R}^{\prime}}\right],
$$

where

$$
\begin{aligned}
\chi(\mathbf{R})= & \frac{-i m \omega_{0}^{2}}{4 \pi^{2} \rho c_{T}^{2}} \int d^{2} k e^{i \mathbf{k} \cdot \mathbf{R}}\left(\frac{k_{y}^{2}}{k^{2} p_{T}}+\frac{\omega^{2} k_{x}^{2} p_{T}}{c_{T}^{2} k^{2} P}\right) \\
& \times\left[\frac{\sin \left(k_{x} L_{x} / 2\right)}{\left(k_{x} L_{x} / 2\right)} \frac{\sin \left(k_{y} L_{y} / 2\right)}{\left(k_{y} L_{y} / 2\right)}\right]^{2}(\| \text { mode }) .
\end{aligned}
$$

In a similar way one can derive the $\chi$ function for a perpendicular frustrated translation:

$$
\begin{aligned}
\chi(\mathbf{R})= & \frac{-i m \omega_{0}^{2}}{4 \pi^{2} \rho c_{T}^{2}} \int d^{2} k e^{i \mathbf{k} \cdot \mathbf{R}} \frac{\omega^{2} p_{L}}{c_{T}^{2} P} \\
& \times\left[\frac{\sin \left(k_{x} L_{x} / 2\right)}{\left(k_{x} L_{x} / 2\right)} \frac{\sin \left(k_{y} L_{y} / 2\right)}{\left(k_{y} L_{y} / 2\right)}\right]^{2}(\perp \text { mode })
\end{aligned}
$$

From here on we will for simplicity denote $\bar{u}$ with $u$. Equations (1), (10), and (11) form the basis for the following discussion.

\section{SINGLE ADSORBATE}

For a single adsorbate Eqs. (1) and (10) take the form

$$
-m \omega^{2} s=-m \omega_{0}^{2}(s-u)+F
$$

and

$$
u=\chi_{0}(u-s),
$$

where $s=s_{\mathbf{0}}, u=u_{1}(\mathbf{0}, 0)$ and $\chi_{0}=\chi(\mathbf{0})$. Combining these equations gives

$$
-\omega^{2} s+\frac{\omega_{0}^{2}}{1-\chi_{0}} s=\frac{F}{m} .
$$

Now $\chi_{0}=\chi_{R}+i \chi_{I}$ is complex and we will show below that $-\chi_{I} \ll 1$, so that

$$
\frac{\omega_{0}^{2}}{1-\chi_{0}} \approx \frac{\omega_{0}^{2}}{1-\chi_{R}}\left(1+\frac{i \chi_{I}}{1-\chi_{R}}\right)=\bar{\omega}_{0}^{2}\left(1+\frac{i \chi_{I}}{1-\chi_{R}}\right),
$$

where $\bar{\omega}_{0}$ is the renormalized resonance frequency. Using Eqs. (11) and writing $\mathbf{k}=\omega_{0} \mathbf{r} / c_{T}, l_{x}=L_{x} \omega / 2 c_{T}$, and $l_{y}$ $=L_{y} \omega_{0} / 2 c_{T}$, and expressing $\mathbf{r}$ in polar coordinates gives

$$
\begin{aligned}
\chi_{0}= & \frac{-i m \omega_{0}^{2} \omega}{4 \pi^{2} \rho c_{T}^{3}} \int_{0}^{r_{c}} d r r \int_{0}^{2 \pi} d \phi\left(\frac{\sin ^{2} \phi}{\left(1-r^{2}\right)^{1 / 2}}\right. \\
& \left.+\frac{\cos ^{2} \phi}{\left(2 r^{2}-1\right)^{2}\left(1-r^{2}\right)^{-1 / 2}+4 r^{2}\left[\left(c_{T} / c_{L}\right)^{2}-r^{2}\right]^{1 / 2}}\right) \\
& \times\left[\frac{\sin \left(l_{x} r \cos \phi\right) \sin \left(l_{y} r \sin \phi\right)}{l_{x} l_{y} r^{2} \cos \phi \sin \phi}\right]^{2}
\end{aligned}
$$

with the cutoff $r_{c}=c_{T} k_{c} / \omega \approx \omega_{c} / \omega$. For point adsorbates (i.e., $l_{x}, l_{y} \rightarrow 0$ ) the cutoff $r_{c}$ is necessary in order for the real part of the integral (14) to converge. Thus, for small adsorbates, an accurate calculation of the real part of $\chi_{0}$ requires a treatment beyond the elastic continuum model (i.e., using an atomistic model for the substrate), but in the present study we do not need to know the numerical value of $\operatorname{Re} \chi_{0}$, since we take the renormalized resonance frequency $\bar{\omega}_{0}$ [see Eq. (13)] directly from the experimental observations. It is interesting to note, however, that there is no need for a cutoff for an adsorbed monolayer film (see Sec. IV). The imaginary part of $\chi_{0}$ is, in general, independent of $r_{c}$ and is accurately given by the elastic continuum model if $\omega_{0} \ll \omega_{c}$. Let us write

$$
\operatorname{Im} \chi_{0}=-\frac{m \omega_{0}^{2} \omega}{8 \pi \rho c_{T}^{3}} \xi_{\|}
$$

where

$$
\begin{aligned}
\xi_{\|}= & \frac{2}{\pi} \operatorname{Re} \int_{0}^{\infty} d r r \int_{0}^{2 \pi} d \phi\left(\frac{\sin ^{2} \phi}{\left(1-r^{2}\right)^{1 / 2}}\right. \\
& \left.+\frac{\cos ^{2} \phi}{\left(2 r^{2}-1\right)^{2}\left(1-r^{2}\right)^{-1 / 2}+4 r^{2}\left[\left(c_{T} / c_{L}\right)^{2}-r^{2}\right]^{1 / 2}}\right) \\
& \times\left[\frac{\sin \left(l_{x} r \cos \phi\right) \sin \left(l_{y} r \sin \phi\right)}{l_{x} l_{y} r^{2} \cos \phi \sin \phi}\right]^{2} .
\end{aligned}
$$

Substituting Eq. (15) into Eq. (13) gives

$$
\frac{\omega_{0}^{2}}{1-\chi_{0}}=\bar{\omega}_{0}^{2}\left(1-i \frac{m \bar{\omega}_{0}^{2} \omega}{8 \pi \rho c_{T}^{3}} \xi_{\|}\right)=\bar{\omega}_{0}^{2}-i \omega \eta_{\|},
$$

with 


$$
\eta_{\|}=\frac{m \bar{\omega}_{0}^{4}}{8 \pi \rho c_{T}^{3}} \xi_{\|}
$$

Substituting Eq. (18) into Eq. (12) gives

$$
-\omega^{2} s+\bar{\omega}_{0}^{2} s-i \omega \eta_{\|} s=F / m
$$

or

$$
m \ddot{s}+m \bar{\omega}_{0}^{2} s+m \eta_{\|} \dot{s}=F .
$$

Hence the coupling to the substrate has two effects: it renormalizes the resonance frequency $\omega_{0} \rightarrow \bar{\omega}_{0}$ and it introduces a damping term in the equation of motion for $s$. The damping is due to the lattice waves radiated into the solid from the oscillating adsorbate (see Fig. 2).

In a similar way one can derive the following equations for the damping of perpendicular adsorbate vibrations:

$$
m \ddot{s}+m \bar{\omega}_{0}^{2} s+m \eta_{\perp} \dot{s}=F,
$$

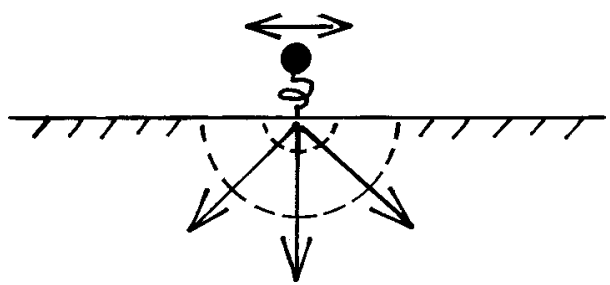

FIG. 2. Damping of a parallel (or perpendicular) adsorbate vibration via emission of elastic waves (one-phonon process).

with

$$
\eta_{\perp}=\frac{m \bar{\omega}_{0}^{4}}{8 \pi \rho c_{T}^{3}} \xi_{\perp}
$$

and

$$
\xi_{\perp}=\frac{2}{\pi} \operatorname{Re} \int_{0}^{\infty} d r r \int_{0}^{2 \pi} d \phi \frac{1}{\left(2 r^{2}-1\right)^{2}\left[\left(c_{T} / c_{L}\right)^{2}-r^{2}\right]^{-1 / 2}+4 r^{2}\left(1-r^{2}\right)^{1 / 2}}\left[\frac{\sin \left(l_{x} r \cos \phi\right) \sin \left(l_{y} r \sin \phi\right)}{l_{x} l_{y} r^{2} \cos \phi \sin \phi}\right]^{2} .
$$

In order to make the expressions (16) and (23) well defined one must add an infinitesimal purely imaginary term $i \epsilon$ (where $\epsilon$ is positive) to the arguments of the square-root functions and, in addition, an infinitesimal positive term $\epsilon^{\prime}$ to the denominator in the term containing $\cos ^{2} \phi$ in Eq. (14) and the similar denominator in Eq. (23). The latter term is necessary in order to make the pole contribution [which corresponds to emission of surface (Rayleigh) phonons] well defined. The sign of $\epsilon$ and $\epsilon^{\prime}$ (both positive) is determined by an analysis where the interaction is turned on slowly from 0 at $t=-\infty$. In the numerical integration of Eqs. (16) and (23) $\epsilon$ and $\epsilon^{\prime}$ are, of course, chosen as small (but not infinitesimal) positive numbers. We note that when $l_{x}=l_{y}=0$, $\xi_{\perp} \approx \xi_{\|} \approx 3.2$, and that the contribution from emission of Rayleigh phonons constitutes about $15 \%$ of $\xi_{\|}$and $64 \%$ of $\xi_{\perp}$ (see Ref. 6).

Walkup et $a l .{ }^{14}$ have presented a very simple (approximate) derivation of Eq. (22): In a simplified picture one regards each adsorbate oscillation as a collision with the substrate, with an energy transferred to the substrate of $\Delta E$ $\approx E\left(\mathrm{~m} / \mathrm{m}_{\mathrm{s}}\right)$, where $E$ is the energy of the adsorbate vibration and $m_{s}$ the "effective" mass of the substrate. During the collision time $\tau \approx 1 / \omega_{0}$ the resulting substrate displacement field extends a distance $c_{T} \tau=c_{T} / \omega_{0}$ into the solid. The effective mass $m_{s}$ is simply the mass contained in the volume element $\Delta V \sim\left(c_{T} / \omega_{0}\right)^{3}$ and thus $m_{s} \sim \rho\left(c_{T} / \omega_{0}\right)^{3}$. Since collisions with the substrate occur with the frequency $\nu$ $=\omega_{0} / 2 \pi$, the net rate of energy transfer per unit time is $d E / d t \approx-\left(\omega_{0} / 2 \pi\right)\left(\mathrm{m} / \mathrm{m}_{\mathrm{s}}\right) E$. This results in an exponential decay of the energy, characterized by the damping constant $\left(\omega_{0} / 2 \pi\right)\left(m / m_{s}\right)=m \omega_{0}^{4} /\left(2 \pi \rho c_{T}^{3}\right)$ which agrees with Eq. (22) except for a factor of order unity.
Note that $\xi_{\|}$and $\xi_{\perp}$ only depend on $l_{x}, l_{y}$, and $c_{T} / c_{L}$. In Fig. 3 we show $\xi_{\|}$and $\xi_{\perp}$ for $c_{T} / c_{L}=0.5$ (typical for metals) as a function of $l_{x}$ for a few different $l_{y}$. As an example, if we approximate $n$-nonane $\left(\mathrm{C}_{9} \mathrm{H}_{20}\right)$ as a rectangular plate with length $L_{x}=14 \AA$ and with width $L_{y}=3 \AA$, we have, for the perpendicular vibrations on $\mathrm{Cu}(100), \omega_{0} \approx 6.9 \mathrm{meV}$, so that $l_{x}=L_{x} \omega_{0} / 2 c_{T} \approx 3.4$ and $l_{y} \approx 0.7$. Using Fig. 3 gives $\xi_{\perp} \approx 1.1$ which is smaller than the $l_{x}=l_{y}=0$ limit by a factor of $\sim 3$.

\section{ORDERED COMMENSURATE ADSORBATE LAYERS}

Bruch and Hansen ${ }^{8}$ and Lewis et al. ${ }^{9,10}$ have calculated the damping of the $\mathbf{q}=\mathbf{0}$ collective adsorbate vibration under the assumption that the adsorbate unit cell is so small that for all (nonvanishing) reciprocal lattice vectors $\mathbf{G}$ (associated with the adsorbate lattice), $G>\omega_{0} / c_{T}$. We first show how these results can be derived using a much simpler approach. When the adsorbate lattice constant $a<\lambda$, where $\lambda$ $=2 \pi c_{T} / \omega_{0}$ is the wavelength of the emitted phonons, we can, when calculating the damping of the $\mathbf{q}=\mathbf{0}$ adsorbate phonon, replace the nonuniform surface stress distribution (2) [or (3)] with an average (uniform) distribution

$$
\sigma_{13}=n_{a} m \omega_{0}^{2}\left[u_{1}(0)-s\right],
$$

where $n_{a}$ is the number of adsorbates per unit area, and where we have used the fact that a uniform tangential surface stress gives rise to a displacement field $u_{1}(z)$, which is polarized parallel to the surface (in the direction of the vibrational displacement coordinate $s$ ) and independent of $x$ and $y$ 

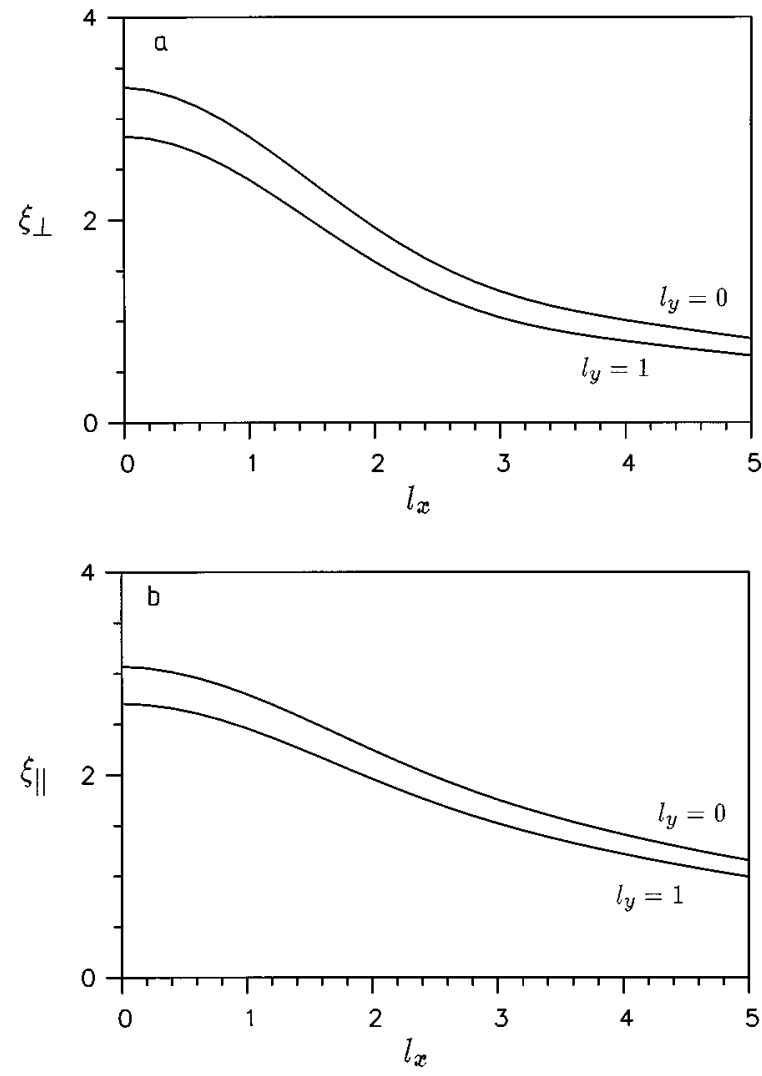

FIG. 3. The dependence of the friction parameters $\xi_{\|}$and $\xi_{\perp}$ on the size of the "adsorbate."

(the emitted elastic waves propagate perpendicular to the surface into the elastic half space); see Fig. 4(a). The elastic displacement field is given by

$$
u_{1}=u_{0} e^{i\left(\omega z / c_{T}-\omega t\right)} .
$$

The boundary condition

$$
\rho c_{T}^{2} \frac{\partial u_{1}}{\partial z}=\sigma_{13} \text { for } z=0
$$

gives

$$
i \rho c_{T} \omega u_{0}=n_{a} m \omega_{0}^{2}\left(u_{0}-s\right)
$$

or
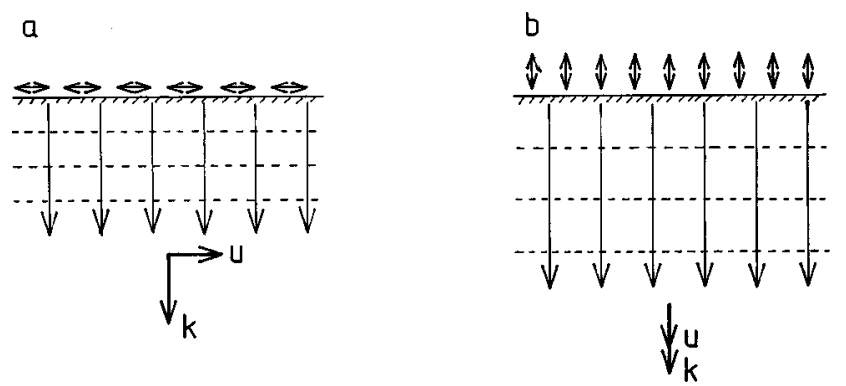

FIG. 4. Elastic waves emitted from a uniform (in space) and oscillating (in time) surface stress distribution, acting (a) parallel and (b) perpendicular to the surface. The emitted elastic waves are transverse in case (a) and longitudinal in case (b), and in both cases the waves propagate orthogonal to the surface into the elastic half space.

$$
u_{0}-s=\frac{i \rho c_{T} \omega s}{n_{a} m \omega_{0}^{2}-i \rho c_{T} \omega} .
$$

Substituting this in Eq. (1) gives

$$
\omega^{2} s=\frac{\omega_{0}^{2} s}{1-n_{a} m \omega_{0}^{2} / i \rho c_{T} \omega} .
$$

Since $n_{a} m \omega_{0}^{2} / \rho c_{T} \omega$ is in general very small for $\omega \approx \omega_{0}$, we get from Eq. (29) for $\omega$ close to the resonance frequency $\omega_{0}$

$$
-m \omega^{2} s+m \omega_{0}^{2}\left(1-i n_{a} m \omega_{0} / \rho c_{T}\right) s=0 .
$$

Thus, the damping

$$
\eta_{\|}=m \omega_{0}^{2} n_{a} / \rho c_{T},
$$

which is identical to the result obtained by Bruch and Hansen $^{8}$ and Lewis et al..$^{9,10}$

A similar calculation as presented above can be performed for the $\mathbf{q}=\mathbf{0}$ perpendicular adsorbate vibration. In this case the displacement field in the solid is polarized perpendicular to the surface and is again independent of $x$ and $y$ (the emitted elastic waves propagate perpendicular to the surface into the elastic half space); see Fig. 4(b). The boundary condition at the surface $z=0$ now takes the form

$$
\rho c_{L}^{2} \frac{\partial u_{3}}{\partial z}=\sigma_{33} \text { for } z=0
$$

where

$$
u_{3}=u_{0} e^{i\left(\omega z / c_{L}-\omega t\right)} .
$$

These equations are similar to Eqs. (26) and (27), except that $c_{T}$ is replaced with $c_{L}$. Thus we get immediately

$$
\eta_{\perp}=m \omega_{0}^{2} n_{a} / \rho c_{L} .
$$

Equations (31) and (32) are valid also for anisotropic elastic media, if $c_{T}$ and $c_{L}$ are interpreted as the sound velocity for an elastic wave propagating in the $z$ direction and polarized along the $x$ and $z$ directions, respectively.

Since typically $c_{L} \approx 2 c_{T}$, one expects roughly twice as large damping for the $\mathbf{q}=\mathbf{0}$ parallel vibration, when compared to that of the perpendicular vibration, assuming that the resonance frequency $\omega_{0}$ is the same. In this context we note that in a recent paper Fuhrmann and $\mathrm{Wöll}^{15}$ presented experimental data for the damping of perpendicular vibrations in $n$-alkanes on various surfaces, and compared the results with the prediction (31) for the damping rate of parallel vibrations (only this formula was known to Fuhrman and Wöll, and they assumed that it is valid also for the perpendicular vibrations). They found that the theory gave a factor of 2 larger damping rates than was deduced from the experiments. If the correct formula (32) is used, the theory is in good agreement with the experiments (see Sec. VI).

Based on Eq. (31) it is easy to estimate the damping of a localized (isolated) adsorbate vibration. The following derivation is of interest not only because of its simplicity [as compared with the derivation of Eq. (18)], but also because of the physical insight it gives. To estimate the damping of an isolated adsorbate, note first that for an ordered adsorbate layer with the Brillouin zone area $A_{\mathrm{BZ}}=(2 \pi)^{2} n_{a}$, only the 


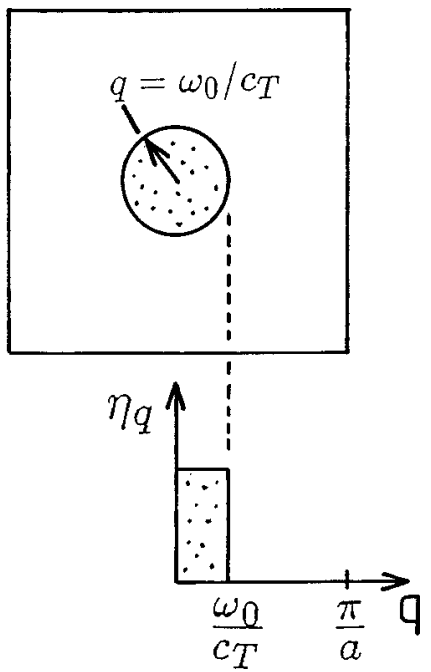

FIG. 5. (a) The circular dotted area (radius $q=\omega_{0} / c_{T}$ ) denotes the region of the adsorbate Brillouin zone (assumed to be a square) where an adsorbate vibration can decay by emission of one bulk phonon. For $q>\omega_{0} / c_{T}$ both energy and momentum cannot be conserved in a one-phonon emission process, and the damping vanishes. (b) Schematic picture of the damping rate as a function of $q$ used for the estimate of the damping rate of an isolated adsorbate vibration (see text). In reality, the damping rate is not constant for $0<q<\omega_{0} / c_{T}$; see Fig. 6 .

collective adsorbate modes with wave vector $q<\omega_{0} / c_{T}$ are damped via one-phonon emission. The point is that bulk phonons [wave vector $\mathbf{k}=\left(\mathbf{q}, k_{z}\right)$ ] with $q>\omega_{0} / c_{T}$ have the energy $c_{T}\left(q^{2}+k_{z}^{2}\right)^{1 / 2}>\omega_{0}$ independent of the value $k_{z}$ of the $z$ component of the (bulk) phonon wave vector. Thus only for a fraction $\pi\left(\omega_{0} / c_{T}\right)^{2} / A_{\mathrm{BZ}}$ of the modes in the adsorbate $\mathrm{BZ}$ will the one-phonon decay process be possible; see Fig. 5(a). Now, roughly speaking, a localized adsorbate vibration can be obtained by summing over all $\mathbf{q}$ modes. If we assume that all the $\mathbf{q}$ modes in the region $q<\omega_{0} / c_{T}$ have the same damping as for the $\mathbf{q}=\mathbf{0}$ mode [given by Eq. (31)], see Fig. $5(\mathrm{~b})$, then the damping of the localized mode becomes

$$
\begin{aligned}
\eta & =\left\langle\eta_{\mathbf{q}}\right\rangle=\frac{1}{A_{\mathrm{BZ}}} \int_{\mathrm{BZ}} d^{2} q \eta_{\mathbf{q}} \\
& \approx\left[\frac{m \omega_{0}^{2} n_{a}}{\rho c_{T}}\right]\left[\frac{\pi\left(\omega_{0} / c_{T}\right)^{2}}{(2 \pi)^{2} n_{a}}\right]=\frac{m \omega_{0}^{4}}{4 \pi \rho c_{T}^{3}},
\end{aligned}
$$

which agrees with Eq. (18) to within a factor of $\xi_{\|} / 2 \approx 1.6$.

Let us now consider the damping of a collective adsorbate mode with arbitrary wave vector $\mathbf{q}$. In addition to the (indirect) interaction between the adsorbates mediated by the elastic solid, we assume that there is an adsorbate-adsorbate interaction which is of nonphononic nature, i.e., one mediated by the substrate electrons (for fixed positions of the substrate ions) or even a direct interaction resulting from, e.g., Pauli repulsion. Inelastic helium scattering has shown that such an additional interaction occurs for the $c(2 \times 2)$ $\mathrm{CO}$ overlayer on $\mathrm{Cu}(100) .{ }^{16}$ In this case Eqs. (1) and (10) take the form

$$
m \ddot{s_{\mathbf{R}}}=\sum_{\mathbf{R}^{\prime}} K\left(\mathbf{R}-\mathbf{R}^{\prime}\right) s_{\mathbf{R}^{\prime}}-m \omega_{0}^{2}\left[s_{\mathbf{R}}-u_{1}(\mathbf{R}, 0)\right]+F_{\mathbf{R}},
$$

with

$$
u_{1}(\mathbf{R}, 0)=\sum_{\mathbf{R}^{\prime}} \chi\left(\mathbf{R}-\mathbf{R}^{\prime}\right)\left[u_{1}\left(\mathbf{R}^{\prime}, 0\right)-s_{\mathbf{R}^{\prime}}\right] .
$$

Let us write

$$
\begin{gathered}
s_{\mathbf{R}}=\sum_{\mathbf{q}} s_{\mathbf{q}} e^{i \mathbf{q} \cdot \mathbf{R}}, \\
s_{\mathbf{q}}=\frac{1}{N} \sum_{\mathbf{R}} s_{\mathbf{R}} e^{-i \mathbf{q} \cdot \mathbf{R}},
\end{gathered}
$$

where $N$ is the number of lattice sites. Substituting Eq. (35) into Eqs. (33) and (34) gives

$$
-m \omega^{2} s_{\mathbf{q}}-\widetilde{K}(\mathbf{q}) s_{\mathbf{q}}=-m \omega_{0}^{2}\left[s_{\mathbf{q}}-u_{1}(\mathbf{q}, 0)\right]+F_{\mathbf{q}},
$$

with

$$
u_{1}(\mathbf{q}, 0)=\tilde{\chi}(\mathbf{q})\left[u_{1}(\mathbf{q}, 0)-s_{\mathbf{q}}\right]
$$

and

$$
\tilde{\chi}(\mathbf{q})=\sum_{\mathbf{R}} \chi(\mathbf{R}) e^{-i \mathbf{q} \cdot \mathbf{R}},
$$

and a corresponding expression for $\widetilde{K}(\mathbf{q})$. Note that since

$$
\sum_{\mathbf{R}} e^{i(\mathbf{k}-\mathbf{q}) \cdot \mathbf{R}}=N \sum_{\mathbf{G}} \delta_{\mathbf{G}, \mathbf{k}-\mathbf{q}} \rightarrow(2 \pi)^{2} n_{a} \sum_{\mathbf{G}} \delta(\mathbf{G}+\mathbf{q}-\mathbf{k}),
$$

where $\{\mathbf{G}\}$ is the reciprocal lattice to $\{\mathbf{R}\}$, we get from Eqs. (11) and (39)

$$
\tilde{\chi}(\mathbf{q})=\sum_{\mathbf{G}} \bar{\chi}(\mathbf{q}+\mathbf{G})
$$

where

$$
\begin{aligned}
\bar{\chi}(\mathbf{k})= & \frac{-i m \omega_{0}^{2} n_{a}}{\rho c_{T}^{2}}\left(\frac{k_{y}^{2}}{k^{2} p_{T}}+\frac{\omega^{2} k_{x}^{2} p_{T}}{c_{T}^{2} k^{2} P(k)}\right) \\
& \times\left[\frac{\sin \left(k_{x} L_{x} / 2\right)}{\left(k_{x} L_{x} / 2\right)} \frac{\sin \left(k_{y} L_{y} / 2\right)}{\left(k_{y} L_{y} / 2\right)}\right]^{2}(\| \text { mode }),
\end{aligned}
$$

where $n_{a}=N / A$ is the number of adsorbates per unit area.

Combining Eqs. (37) and (38) gives

$$
-\omega^{2} s_{\mathbf{q}}-\frac{\widetilde{K}(\mathbf{q})}{m} s_{\mathbf{q}}+\frac{\omega_{0}^{2}}{1-\tilde{\chi}(\mathbf{q})} s_{\mathbf{q}}=\frac{F_{\mathbf{q}}}{m}
$$

or

$$
s_{\mathbf{q}}=\frac{F_{\mathbf{q}} / m}{-\omega^{2}-[\widetilde{K}(\mathbf{q}) / m]+\omega_{0}^{2} /[1-\tilde{\chi}(\mathbf{q})]} .
$$

Since $|\tilde{\chi}| \ll 1$ (see below), we can expand $1 /(1-\tilde{\chi}) \approx 1+\tilde{\chi}$. Next, if we define 


$$
\omega_{\mathbf{q}}^{2}=\omega_{0}^{2}[1+\operatorname{Re} \tilde{\chi}(\mathbf{q})]-\widetilde{K}(\mathbf{q}) / m
$$

and

$$
\eta_{\mathbf{q}}(\omega)=-\omega_{0}^{2} \operatorname{Im} \tilde{\chi}(\mathbf{q}) / \omega,
$$

then Eq. (42) takes the form

$$
s_{\mathbf{q}}=\frac{F_{\mathbf{q}} / m}{\omega_{\mathbf{q}}^{2}-\omega^{2}-i \omega \eta_{\mathbf{q}}} .
$$

Thus, the vibrational profile will be an approximate Lorentzian, centered at $\omega=\omega_{\mathbf{q}}$, and with the full width at half maximum equal to $\eta_{\mathbf{q}}\left(\omega_{\mathbf{q}}\right)$. Let us now consider the $\mathbf{q}=\mathbf{0}$ limit, which can be probed using, e.g., inelastic helium scattering. If, as is usually the case, $G>\omega_{0} / c_{T}$ for all nonvanishing $\mathbf{G}$ (see below), only the $\mathbf{G}=\mathbf{0}$ term in Eq. (40) will contribute to $\operatorname{Im} \tilde{\chi}(\mathbf{0})$. Thus we get

$$
\operatorname{Im} \tilde{\chi}(\mathbf{0})=-\frac{m \omega_{0}^{2} n_{a}}{\rho c_{T} \omega} .
$$

Since $\widetilde{K}(\mathbf{0})=0$, and neglecting the small term $\operatorname{Re} \tilde{\chi}(\mathbf{0})$ in Eq. (43), we get $\omega_{\mathbf{q}=\mathbf{0}} \approx \omega_{0}$ and thus $\eta_{\mathbf{q}=\mathbf{0}} \approx m \omega_{0}^{2} n_{a} / \rho c_{T}$, which agrees with the result derived earlier using a much simpler procedure. The present derivation shows in addition, however, that in the $\mathbf{q}=\mathbf{0}$ limit, the damping is independent of the size of the adsorbates. Let us now briefly discuss how the damping $\eta_{\mathbf{q}}$ depends on $\mathbf{q}$. We note first that the average of $\tilde{\chi}(\mathbf{q})$ over the Brillouin zone gives

$$
\begin{aligned}
\frac{1}{A_{\mathrm{BZ}}} \int_{\mathrm{BZ}} d^{2} q \tilde{\chi}(\mathbf{q}) & =\frac{1}{A_{\mathrm{BZ}}} \int_{\mathrm{BZ}} d^{2} q \sum_{\mathbf{G}} \bar{\chi}(\mathbf{q}+\mathbf{G}) \\
& =\frac{1}{A_{\mathrm{BZ}}} \int d^{2} q \bar{\chi}(\mathbf{q})=\chi(\mathbf{0}),
\end{aligned}
$$

where the last equality follows by comparing Eq. (41) with Eq. (11a) and using that $A_{\mathrm{BZ}}=(2 \pi)^{2} n_{a}$. Thus, if we neglect the contribution to $\omega_{\mathbf{q}}$ from $\operatorname{Re} \tilde{\chi}(\mathbf{q})$ (this contribution is usually rather small; see below) and assume that $\widetilde{K}(\mathbf{q})$ is small (usually the case), then the average of $\eta_{\mathbf{q}}$ over the Brillouin zone is identical to the damping $\eta$ of an isolated adsorbate calculated at the frequency $\omega_{0}$. Since the expressions for the damping of the $\mathbf{q}=\mathbf{0}$ collective mode and that for the damping of an isolated adsorbate often give very different numerical values for the damping, it follows as a corollary that $\eta_{\mathbf{q}}$ varies strongly with $\mathbf{q}$ in the Brillouin zone. This is in accordance with Fig. 6 where we show $\eta_{\mathbf{q}}$ as a function of $q$ for both the parallel and perpendicular adsorbate vibrations for $l_{x}=l_{y}=0$. The functions shown are $\zeta_{\|}(q)$ and $\zeta_{\perp}(q)$ defined by

$$
\begin{gathered}
\eta_{\mathbf{q}}=\frac{m \omega_{0}^{2} n_{a}}{\rho c_{T}} \zeta_{\|}(q) \text { (for parallel vibrations), } \\
\eta_{\mathbf{q}}=\frac{m \omega_{0}^{2} n_{a}}{\rho c_{L}} \zeta_{\perp}(q) \text { (for perpendicular vibrations). }
\end{gathered}
$$

Since for $\mathbf{q}=\mathbf{0}$ these formulas must reduce to Eqs. (31) and (32), respectively, we must have $\zeta_{\|}(0)=\zeta_{\perp}(0)=1$. Using
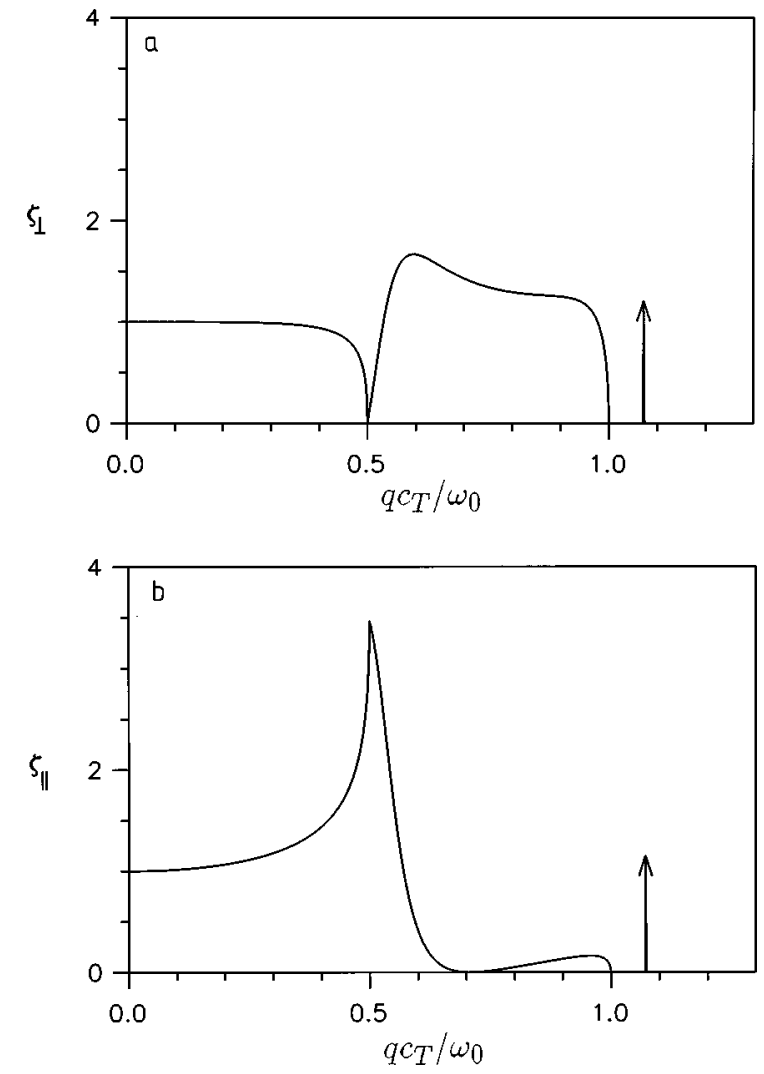

FIG. 6. The quantities $\zeta_{\|}(q)$ and $\zeta_{\perp}(q)$ are proportional to the damping of parallel and perpendicular collective q-adsorbate modes, respectively. See the text for definitions.

inelastic helium scattering it should be possible to study the q dependence of $\eta_{\mathbf{q}}$; see Sec. VI.

Finally, note that

$$
\begin{aligned}
\operatorname{Re} \tilde{\chi}(\mathbf{0}) \approx & \sum_{\mathbf{G} \neq \mathbf{0}} \frac{m \omega_{0}^{2} n_{a}}{\rho c_{T}^{2} G}\left(\frac{G_{y}^{2}}{G^{2}}+\frac{G_{x}^{2}}{2 G^{2}\left(1-c_{T}^{2} / c_{L}^{2}\right)}\right) \\
& \times\left[\frac{\sin \left(G_{x} L_{x} / 2\right) \sin \left(G_{y} L_{y} / 2\right)}{\left(G_{x} L_{x} / 2\right)\left(G_{y} L_{y} / 2\right)}\right]^{2} .
\end{aligned}
$$

In most cases this formula gives $\operatorname{Re} \tilde{\chi}(\mathbf{0}) \ll 1$ so that $\omega_{\mathbf{q}}$ [see Eq. (43)], for $\mathbf{q}=\mathbf{0}$, is usually close to $\omega_{0}$.

The calculations presented above can be repeated for perpendicular adsorbate vibrations and give exactly the same results, except that $\bar{\chi}(\mathbf{k})$ now takes the form

$$
\begin{aligned}
\bar{\chi}(\mathbf{k})= & \frac{-i m \omega_{0}^{2} n_{a}}{\rho c_{T}^{2}} \frac{\omega^{2} p_{L}}{c_{T}^{2} P(k)} \\
& \times\left[\frac{\sin \left(k_{x} L_{x} / 2\right)}{\left(k_{x} L_{x} / 2\right)} \frac{\sin \left(k_{y} L_{y} / 2\right)}{\left(k_{y} L_{y} / 2\right)}\right]^{2}(\perp \text { mode }) .
\end{aligned}
$$

\section{DISORDERED COMMENSURATE ADSORBATE LAYERS}

Let us consider a disordered adsorbate layer. Assume that the adsorbates are randomly distributed on a twodimensional (2D) lattice (lattice gas); see Fig. 7. Let $n_{\mathbf{R}}=1$ if lattice site $\mathbf{R}$ is occupied and zero otherwise. Thus the cov- 


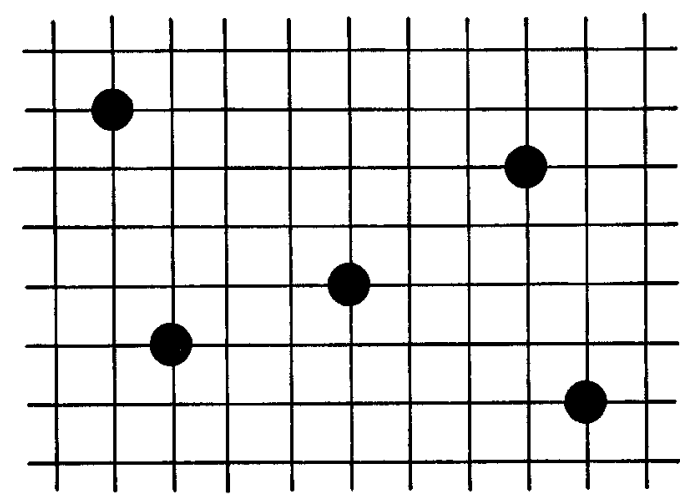

FIG. 7. A lattice gas model of an adsorbate layer.

erage $\theta=\left\langle n_{\mathbf{R}}\right\rangle$, where $\langle\cdots\rangle$ stands for ensemble averaging. In the present case Eq. (10) takes the form

$$
u_{1}(\mathbf{R})=\sum_{\mathbf{R}^{\prime}} n_{\mathbf{R}^{\prime}} \chi\left(\mathbf{R}-\mathbf{R}^{\prime}\right)\left[u_{1}\left(\mathbf{R}^{\prime}\right)-s_{\mathbf{R}^{\prime}}\right] .
$$

Let us now define $\chi_{*}(\mathbf{R})=\chi(\mathbf{R})$ for nonvanishing $\mathbf{R}$, while $\chi_{*}(\mathbf{0})=0$. Thus Eq. (49) takes the form

$$
\begin{aligned}
u_{1}(\mathbf{R})= & \chi(\mathbf{0})\left[u_{1}(\mathbf{R})-s_{\mathbf{R}}\right] \\
& +\sum_{\mathbf{R}^{\prime}} n_{\mathbf{R}^{\prime}} \chi_{*}\left(\mathbf{R}-\mathbf{R}^{\prime}\right)\left[u_{1}\left(\mathbf{R}^{\prime}\right)-s_{\mathbf{R}^{\prime}}\right] .
\end{aligned}
$$

We now calculate the ensemble average of $u_{1}(\mathbf{R})$. In the simplest (mean field) approximation [equivalent to the averaged $T$-matrix approximation (ATA) used in band structure calculations for alloys] we simply replace $n_{\mathbf{R}} \rightarrow\left\langle n_{\mathbf{R}}\right\rangle=\theta$ in Eq. (50) to get

$$
u_{1}(\mathbf{R})=\chi(\mathbf{0})\left[u_{1}(\mathbf{R})-s_{\mathbf{R}}\right]+\theta \sum_{\mathbf{R}^{\prime}} \chi_{*}\left(\mathbf{R}-\mathbf{R}^{\prime}\right)\left[u_{1}\left(\mathbf{R}^{\prime}\right)-s_{\mathbf{R}^{\prime}}\right]
$$

or

$$
u_{1}(\mathbf{q})=\chi(\mathbf{0})\left[u_{1}(\mathbf{q})-s_{\mathbf{q}}\right]+\theta \tilde{\chi}_{*}(\mathbf{q})\left[u_{1}(\mathbf{q})-s_{\mathbf{q}}\right],
$$

where

$$
\begin{aligned}
\tilde{\chi}_{*}(\mathbf{q}) & =\sum_{\mathbf{R}} \chi_{*}(\mathbf{R}) e^{-i \mathbf{q} \cdot \mathbf{R}} \\
& =\sum_{\mathbf{R}} \chi(\mathbf{R}) e^{-i \mathbf{q} \cdot \mathbf{R}}-\chi(\mathbf{0}) \\
& =\tilde{\chi}(\mathbf{q})-\chi(\mathbf{0}) .
\end{aligned}
$$

Substituting this into Eq. (51) gives

$$
u_{1}(\mathbf{q})=[(1-\theta) \chi(\mathbf{0})+\theta \tilde{\chi}(\mathbf{q})]\left[u_{1}(\mathbf{q})-s_{\mathbf{q}}\right] .
$$

This is the same result as derived earlier for the ordered adsorbate layer except that $\tilde{\chi}(\mathbf{q}) \rightarrow(1-\theta) \chi(\mathbf{0})+\theta \tilde{\chi}(\mathbf{q})$. Thus, comparing with Eq. (44) it is immediately clear that the damping of the $\mathbf{q}=\mathbf{0}$ parallel adsorbate mode in the present case is given by

$$
\eta_{\mathbf{0}} \approx(1-\theta) \frac{m \omega_{0}^{4} \xi_{\|}}{8 \pi \rho c_{T}^{3}}+\frac{m \omega_{0}^{2} n_{a}}{\rho c_{T}},
$$

where $n_{a}=\theta n$ and $n$ is the number of binding sites per unit surface area. The theory above can, if necessary, be improved by using the coherent potential approximation (CPA) instead of the ATA. The first term in Eq. (53) is the incoherent contribution to the damping while the second term, which depends on the adsorbate coverage, is the coherent contribution. As an application, consider $3 \%$ of a monolayer of $\mathrm{CO}$ on $\mathrm{Cu}(100)$, which recently has been studied using inelastic helium atom scattering. ${ }^{17}$ The lifetime of the parallel frustrated translation was measured to be $8 \pm 1$ ps. Using the observed resonance frequency $\omega_{0} \approx 32 \mathrm{~cm}^{-1}$, Eq. (53) gives $8 \mathrm{ps}$, in good agreement with experiment. The contributions from the first and second terms in Eq. (53) are $1 /(10.5 \mathrm{ps})$ and $1 /(29.4 \mathrm{ps})$, respectively; so in this case the damping is dominated by the incoherent contribution. In the calculation we have used the $\mathrm{Cu}$ mass density $\rho$ $=8936 \mathrm{~kg} / \mathrm{m}^{3}$ and, for the second term in Eq. (53), $c_{T}$ $=2899 \mathrm{~m} / \mathrm{s}$, which is the sound velocity for a transverse elastic wave propagating perpendicular to the $\mathrm{Cu}(100)$ plane, while in the first term in Eq. (53) we have used the "average" transverse sound velocity $c_{T}=2270 \mathrm{~m} / \mathrm{s}$, since in this case elastic waves are emitted in all directions and, furthermore, the derivation of the first term in Eq. (53) is based on the assumption of an isotropic elastic media.

\section{COMPARISON WITH EXPERIMENTS}

In this section we present a brief summary of an extensive set of experiments for a prototype lubricant molecule, octane, adsorbed on a variety of different materials with strongly different elastic and electronic properties, ranging from hard insulator diamond to soft metal lead. The molecules were adsorbed on the different substrates typically at temperatures of $180 \mathrm{~K}$ by backfilling an ultrahigh-vacuum chamber with the corresponding gas (see Ref. 18 for details on the experiments). In all cases the He-atom angular distributions recorded after deposition indicated the presence of high-quality, well-ordered adlayers on all the substrates studied here. The frustrated translational mode of the adsorbed molecules polarized normal to the surface is located in the far-infrared regime $\left(7.5 \mathrm{meV}\right.$ or $\left.60 \mathrm{~cm}^{-1}\right)$ and has been studied using inelastic scattering of thermal energy He atoms. The intrinsic linewidth of this low-energy vibrational mode can be determined by deconvoluting the experimental timeof-flight spectra with the known apparatus function. ${ }^{18}$ The perpendicular polarized mode was observed for all substrates. For octane adsorbed on the $\mathrm{Cu}(111)$ surface we could also detect the mode polarized parallel to the substrate. ${ }^{15}$ The results for the various adsorbate-substrate combinations are compiled in Table I, where they are compared to theory [Eqs. (31) and (32), using the mass density and sound velocities given in Table II]. It is clear that the theory is in relative good agreement with experiment. Also shown in Table I are the theoretical results for the damping of isolated (independent) adsorbate vibrations, $\eta_{\text {ind }}$, as given by Eqs. (18) and (22). This theory is clearly not applicable in the present case involving the damping of the $\mathbf{q}=\mathbf{0}$ collective phonon mode of well-ordered, densely packed overlayers, where emission 
TABLE I. Experimental determined (extracted from a line shape analysis) energies $\omega_{0}$ and damping $\eta_{0}$ of the $\mathrm{q}=0$ collective frustrated translation normal to the surface $(\perp$ mode), for ordered layers of alkanes adsorbed on different surfaces. Furthermore, the area $A$ per molecule within the monolayer films is listed. The values are compared with predictions for the phononic contribution to the lifetime broadening. Also shown is the theoretically predicted (with $L_{x}=L_{y}=0$ ) damping $\eta_{\text {ind }}$ for the case of isolated (independent) adsorbate vibrations. All experimental data correspond to values at the $\bar{\Gamma}$ point (i.e., $q=0$ ) of the surface Brilluoin zone, with the exception of the $\|$ mode of octane $\left(q=0.52 \AA^{-1}\right)$ and perdeuterated octane on $\mathrm{Cu}(111)(q$ $=0.65 \AA^{-1}$ ).

\begin{tabular}{|c|c|c|c|c|c|c|}
\hline \multirow[b]{2}{*}{ System } & \multirow[b]{2}{*}{ Mode } & \multirow[b]{2}{*}{$\mathrm{A}\left[\AA^{2}\right]$} & \multicolumn{2}{|c|}{ Experiment } & \multicolumn{2}{|c|}{ Theory } \\
\hline & & & $\omega_{0}[\mathrm{meV}]$ & $\eta_{0}[\mathrm{meV}]$ & $\eta_{0}[\mathrm{meV}]$ & $\eta_{\text {ind }}[\mathrm{meV}]$ \\
\hline \multirow[t]{2}{*}{ Octane $/ \mathrm{Cu}(111)$} & $\perp$ & 48.40 & 7.2 & 0.45 & 0.74 & 2.4 \\
\hline & $\|$ & 48.40 & 3.0 & 0.29 & 0.05 & 0.07 \\
\hline \multirow[t]{2}{*}{$d$-octane $/ \mathrm{Cu}(111)$} & $\perp$ & 48.40 & 6.6 & 0.52 & 0.74 & 2.0 \\
\hline & $\|$ & 48.40 & 2.8 & 0.19 & 0.05 & 0.06 \\
\hline Hexane/Cu(111) & $\perp$ & $47.38^{\mathrm{a}}$ & 7.0 & 0.48 & 0.87 & 1.7 \\
\hline Nonane/Cu(111) & $\perp$ & 62.05 & 6.6 & 0.52 & 0.74 & 2.0 \\
\hline Decane/Cu(111) & $\perp$ & $70.31^{\mathrm{a}}$ & 6.6 & 0.52 & 0.74 & 2.0 \\
\hline Hexane/Pb(111) & $\perp$ & $47.38^{\mathrm{a}}$ & 6.7 & 0.34 & (1.3) & $(20.4)$ \\
\hline Octane $/ \mathrm{Pb}(111)$ & $\perp$ & $58.82^{\mathrm{a}}$ & 6.6 & 0.26 & (1.1) & $(25.4)$ \\
\hline$d$-octane/Pb(111) & $\perp$ & $58.82^{\mathrm{a}}$ & 6.2 & 0.32 & $(1.1)$ & $(22.9)$ \\
\hline Octane/C(111) & $\perp$ & 48.10 & 7.0 & 0.38 & 0.35 & 0.03 \\
\hline Decane/C(111) & $\perp$ & $70.31^{\mathrm{a}}$ & 7.0 & 0.32 & 0.36 & 0.04 \\
\hline Octane/Ru(0001) & $\perp$ & $58.82^{\mathrm{a}}$ & 7.3 & 1.12 & 0.32 & 0.32 \\
\hline Octane $/ \mathrm{Ru}(0001) \mathrm{H}(1 \times 1)$ & $\perp$ & $58.82^{\mathrm{a}}$ & 7.6 & 0.42 & 0.35 & 0.38 \\
\hline$C_{6} F_{14} / \mathrm{Cu}(111)$ & $\perp$ & $61.12^{\mathrm{a}}$ & 2.8 & 0.63 & 0.27 & 0.17 \\
\hline$C_{6} F_{14} / \mathrm{Pb}(111)$ & $\perp$ & $61.12^{\mathrm{a}}$ & 2.4 & 0.76 & 0.47 & 1.3 \\
\hline
\end{tabular}

${ }^{a}$ Estimated from the van der Waals radius of the molecule.

of phonons into the substrate is strongly influenced by interference phenomena, which are taken into account in Eqs. (31) and (32).

Note that for diamond there is a huge difference between the damping rates $\eta_{\text {ind }}$ and $\eta_{\mathbf{0}}$, for independent (isolated) and collective (high-coverage) $\mathbf{q}=\mathbf{0}$ adsorbate vibrations, respectively. This difference is related to the high sound velocities of diamond. Since $\eta_{\text {ind }}$ is so small for hydrocarbons on diamond, and since $\eta_{\text {ind }}$ is proportional to the solid-fluid heat transfer coefficient (see Sec. VII), it is clear that in sliding friction with boundary-lubricated diamond surfaces, the lubrication film may heat up to very high temperatures already at low sliding velocities (see Ref. 2).

In the case of octane adsorbed on $\mathrm{Cu}(111)$ the linewidths

TABLE II. Mass densities $\rho$ and the transverse $c_{T}$ and longitudinal $c_{L}$ sound velocities for elastic waves propagating perpendicular to the (111) plane (for $\mathrm{Cu}, \mathrm{Pb}$, and diamond) and perpendicular to the (0001) plane (for $\mathrm{Ru}$ ).

\begin{tabular}{lccc}
\hline \hline Material & $\rho\left[\mathrm{kg} / \mathrm{m}^{3}\right]$ & $c_{T}[\mathrm{~m} / \mathrm{s}]$ & $c_{L}[\mathrm{~m} / \mathrm{s}]$ \\
\hline Copper & 8930 & 2130 & 5150 \\
Lead & 11340 & 860 & 2340 \\
Ruthenium & 12360 & 3850 & 14820 \\
Diamond & 2260 & 25990 & 40080 \\
\hline \hline
\end{tabular}

of the perpendicular mode have been determined as a function of momentum transfer. The corresponding results are shown in Fig. 8(b), together with a structural model of the octane/ $\mathrm{Cu}(111)$ unit cell. Note that for large wave vectors the damping is not zero as expected from the theory presented in Sec. IV, but remains at a finite value. This behavior is different to that seen for Ar adsorbed on $\operatorname{Pt}(111)$, where the intrinsic line width of the Ar vibration normal to the surface dropped to zero for wave vectors larger than $q=\omega_{0} / c_{T}$ $\approx 0.5 \AA^{-1}$ (Ref. 19); see Fig. 8(a). [Compare the experimental data in Fig. 8(a) with theory, Fig. 6(a).] This difference may be related to the fact that in the case of octane the transfer of vibrational energy into the parallel mode (see above) may be more important than in the case of Ar (where the parallel mode could not be observed experimentally ${ }^{19}$ ). Alternatively, it is possible that the residual lifetime broadening of around $0.25 \mathrm{meV}$ in case of octane adsorbed on $\mathrm{Cu}(111)$ has to be assigned to the excitation of $e-h$ pairs. In case of octane adlayers adsorbed on $\mathrm{Ru}(0001)$ this electronic damping has been observed to dominate the phononic damping. ${ }^{5}$ In fact, X-ray-absorption spectroscopy (XAS) reveals that the electronic molecule-substrate coupling, which is present for octane on $\mathrm{Ru}(0001)$, is also present for octane on $\mathrm{Cu}(111) .{ }^{20} \mathrm{~A}$ third possibility is that the defect density in the octane layers is so large that the $q$ conservation breaks down, leading to a $q$-independent background. However, the 


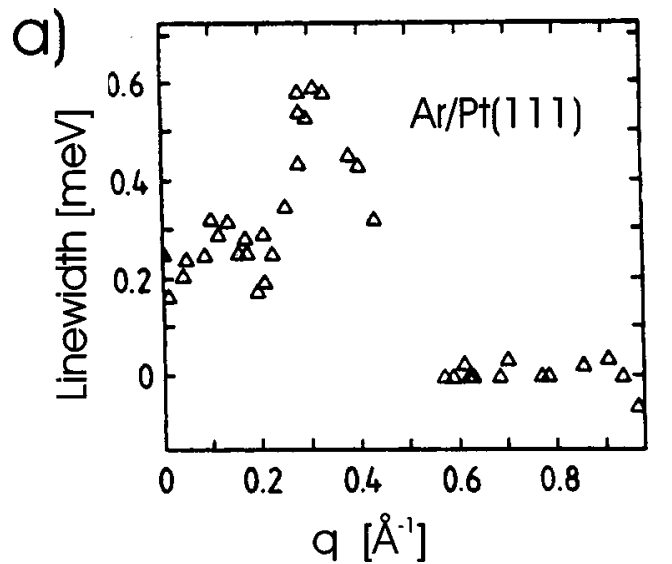

b)

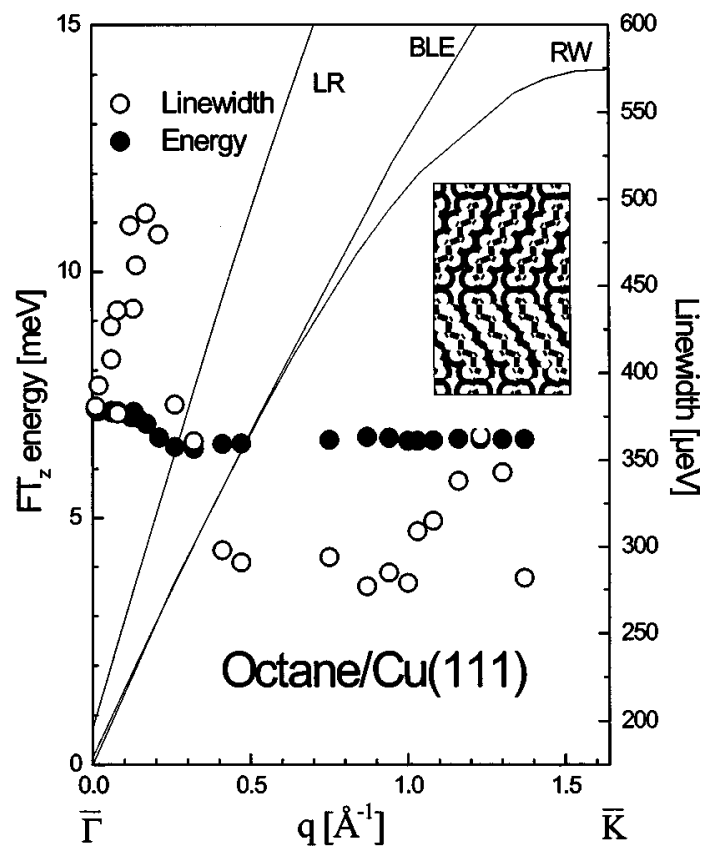

FIG. 8. Intrinsic linewidths of the vibration normal to the surface for two different types of adsorbate systems as determined by inelastic He atom scattering: (a) Data from Zeppenfeld et al. for an Ar monolayer adsorbed on $\operatorname{Pt}(111)$, measured along the $\overline{\Gamma M}$ symmetry direction, from Ref. 19. The $\perp$-mode energy amounts to between 4.8 and $5.1 \mathrm{meV}$. (b) Intrinsic linewidths (right scale) and frequencies (left scale) for the $\perp$ mode of a monolayer of octane adsorbed on $\mathrm{Cu}(111)$ recorded along the $\overline{\Gamma K}$ symmetry direction.

high quality of the He-atom and low-energy electron diffraction (LEED) diffraction patterns recorded for octane adsorbed on $\mathrm{Cu}(111)$ (Ref. 21), and the appreciable size of the residual damping at large wave vectors, do not favor the latter explanation.

\section{DISCUSSION}

We have shown above that the damping $\eta_{\mathbf{q}}$ of the collective adsorbate vibrational mode with wave vector $\mathbf{q}$ depends on $\mathbf{q}$, and that the average of $\eta_{\mathbf{q}}$ over all the wave vectors in the first Brillouin zone gives (approximately) the same damping as experienced of a single (isolated) adsorbate, i.e.,
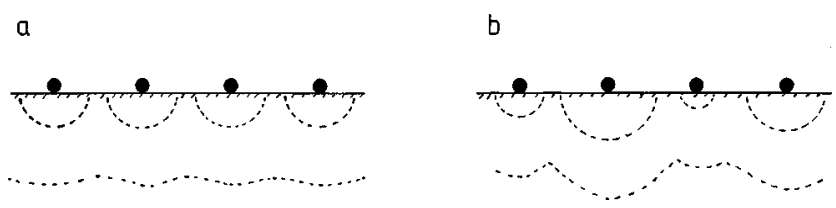

FIG. 9. For short times $t \ll \tau^{*}$ the adsorbates oscillate in phase, while for $t \gg \tau^{*}$ the phases of the oscillators vary randomly with the lateral position. $\tau^{*}$ is the dephasing time.

$$
\left\langle\eta_{\mathbf{q}}\right\rangle=\frac{1}{A_{\mathrm{BZ}}} \int_{\mathrm{BZ}} d^{2} q \eta_{\mathbf{q}} \approx \eta,
$$

where $A_{\mathrm{BZ}}$ is the area of the Brillouin zone, and where $\eta$ is given by Eq. (18) or (22) for parallel and perpendicular adsorbate vibrations, respectively. In general, $\eta_{\mathbf{q}}$ may vary strongly with $\mathbf{q}$, and depending on the type of experiments performed, very different one-phonon damping rates may result. This point is of crucial importance and has been overlooked in earlier studies. Let us consider the problem in greater detail.

The damping of low-frequency adsorbate vibrations can be studied using the inelastic scattering of helium atoms. From these studies one can, in principle, directly obtain the wave-vector-dependent damping $\eta_{\mathbf{q}}$ (see Sec. VI). We note, however, one possible complication in the analysis of the experimental data. If the adsorbate mode under consideration is affected not only by energy relaxation, but also by (pure) dephasing, then the situation can be very complicated. The point is that if the dephasing rate is of order of, or larger than, the energy relaxation rate, then even if the oscillators are excited coherently (say, the $\mathbf{q}=\mathbf{0}$ mode), before the excited state has decayed by energy relaxation, the oscillators will be (partly) out of phase, which in turn will strongly affect the energy relaxation processes; see Fig. 9. In particular, in the limiting case where the dephasing is much faster than the energy relaxation, the oscillators will have nearly random phases and the energy relaxation rate should now be similar to that obtained after averaging $\eta_{\mathbf{q}}$ over all wave vectors $\mathbf{q}$, which is (approximately) given by the damping of an isolated adsorbate (see above). Let us consider this problem in greater detail.

We assume that, as the result of a (pure) dephasing interaction, the relative phases of the adsorbate oscillators change slowly with time [compared to the time dependence of $\left.\cos \left(\omega_{0} t\right)\right]$. In this case the elastic wave emitted from the adsorbate layer will be of the form (we neglect the vector aspect of the field which is of no interest for what follows)

$$
u \approx \sum_{\mathbf{R}} \frac{e^{i\left[k_{0}|\mathbf{x}-\mathbf{R}|-\omega_{0} t-\phi_{\mathbf{R}}(t)\right]}}{|\mathbf{x}-\mathbf{R}|}=\sum_{\mathbf{R}} u_{\mathbf{R}} e^{-i \phi_{\mathbf{R}}(t)},
$$

where the phase $\phi_{\mathbf{R}}$ is determined by

$$
\phi_{\mathbf{R}}(t)=\int_{0}^{t} d t^{\prime} \theta_{\mathbf{R}}\left(t^{\prime}\right)
$$

and $\theta_{\mathbf{R}}(t)$ is a stochastic variable with $\left\langle\theta_{\mathbf{R}}\right\rangle=0$. The fluctuating variable $\theta(t)$ could, e.g., result from anharmonic coupling between the adsorbate mode under consideration and 
other low-frequency vibrational modes (see Ref. 6). We neglect the correlations between $\theta_{\mathbf{R}}$ and $\theta_{\mathbf{R}^{\prime}}$ for $\mathbf{R}$ different from $\mathbf{R}^{\prime}$, and take

$$
\left\langle\theta_{\mathbf{R}}(t) \theta_{\mathbf{R}^{\prime}}\left(t^{\prime}\right)\right\rangle=a \delta_{\mathbf{R R}^{\prime}} \delta\left(t-t^{\prime}\right),
$$

where $a$ is a constant. The radiated intensity is proportional to $\left\langle|u|^{2}\right\rangle$ where $\langle\cdots\rangle$ denotes averaging over different realization of the stochastic variable $\theta$ (which is equivalent to an ensemble average). Using Eq. (54) gives

$$
\left\langle|u|^{2}\right\rangle=\sum_{\mathbf{R}}\left|u_{\mathbf{R}}\right|^{2}\left(1-\left|\left\langle e^{i \phi}\right\rangle\right|^{2}\right)+\left|\sum_{\mathbf{R}} u_{\mathbf{R}}\right|^{2}\left|\left\langle e^{i \phi}\right\rangle\right|^{2} .
$$

The cummulant expansion to second order gives

$$
\left\langle e^{i \phi}\right\rangle=\left\langle 1+i \phi-\phi^{2} / 2+\cdots\right\rangle \approx e^{-\left\langle\phi^{2}\right\rangle / 2},
$$

where we have used that $\langle\phi\rangle=0$. Now, since

$$
\left\langle\phi^{2}(t)\right\rangle=\int_{0}^{t} d t^{\prime} d t^{\prime \prime}\left\langle\theta\left(t^{\prime}\right) \theta\left(t^{\prime \prime}\right)\right\rangle=a t,
$$

Eq. (57) gives the following expression for the radiated intensity:

$$
I=I_{\text {ind }}\left(1-e^{-t / \tau^{*}}\right)+I_{\text {coll }} e^{-t / \tau^{*}},
$$

where $\tau^{*}=1 / a$ is the (pure) dephasing time, $I_{\text {ind }}$ is the emitted intensity for independent (isolated) oscillators, and $I_{\text {coll }}$ the emitted intensity for the $\mathbf{q}=\mathbf{0}$ mode of the collective system of oscillators. We can write

$$
I_{\text {ind }}=\frac{\hbar \omega_{0}}{\tau_{\text {ind }}} \text { and } I_{\text {coll }}=\frac{\hbar \omega_{0}}{\tau_{\text {coll }}},
$$

where $1 / \tau_{\text {ind }}$ is the damping rate for independent oscillators as given by Eq. (18) or (22), while $1 / \tau_{\text {coll }}$ is the damping rate for the $\mathbf{q}=\mathbf{0}$ mode of the collective system of oscillators as given by Eq. (31) or (32). The energy relaxation time $\tau$ can be obtained from the requirement

$$
\int_{0}^{\tau} d t I(t)=\hbar \omega_{0}
$$

Using Eqs. (58) and (59) gives after some simplifications

$$
1=\frac{\tau}{\tau_{\text {ind }}}+\tau^{*}\left(\frac{1}{\tau_{\text {coll }}}-\frac{1}{\tau_{\text {ind }}}\right)\left(1-e^{-\tau / \tau^{*}}\right) .
$$

Figure 10 shows $\tau / \tau_{\text {ind }}$ as a function of $\tau^{*} / \tau_{\text {ind }}$ for $\tau_{\text {coll }} / \tau_{\text {ind }}=2$. Note that, as expected, $\tau$ varies smoothly between $\tau_{\text {ind }}$ for short dephasing times and $\tau_{\text {coll }}$ for long dephasing times. We note that dephasing may result from anharmonic coupling between the frustrated translational modes and thermally excited phonons, or by interaction with thermally excited electron-hole pairs. If a nonzero gas pressure occurs, then a collision between the gas atoms and the adsorbates will also give rise to dephasing. Experiments by Graham et al. have shown that dephasing is very important for the $\mathrm{CO} / \mathrm{Cu}(100)$ adsorbate system, where, at low adsorbate coverage, the linewidth (or damping) of the parallel frustrated translation $\left(\omega_{0} \approx 32 \mathrm{~cm}^{-1}\right)$ increases from 0.7 $\mathrm{cm}^{-1}$ at zero temperature to $1.6 \mathrm{~cm}^{-1}$ at $T=150 \mathrm{~K} .{ }^{17}$ It is

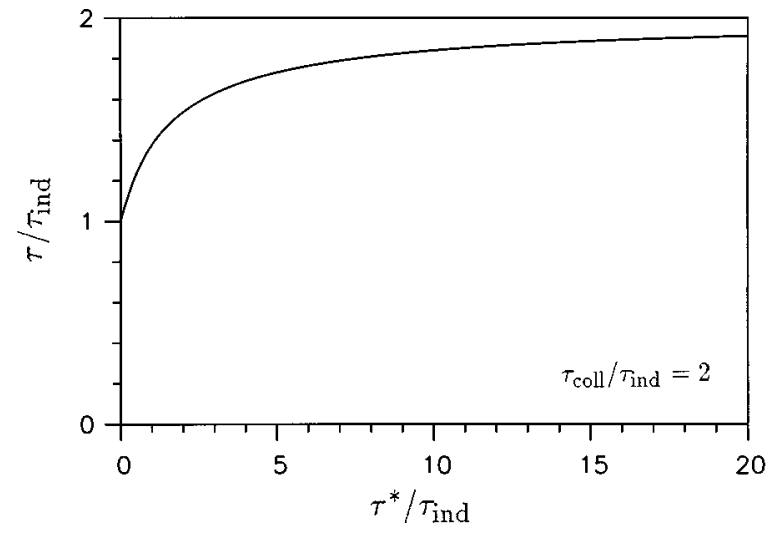

FIG. 10. The variation of the energy relaxation time $\tau$ (due to one-phonon emission) with the (pure) dephasing time $\tau^{*} . \tau_{\text {ind }}$ and $\tau_{\text {coll }}$ denote the energy relaxation time for an isolated (independent) adsorbate vibration and for an ordered layer of coherent oscillating $(\mathbf{q}=\mathbf{0}$ mode $)$ adsorbates. In the calculation we have assumed that $\tau_{\text {coll }}=2 \tau_{\text {ind }}$.

clear that for the ordered $c(2 \times 2) \mathrm{CO}$ layer on $\mathrm{Cu}(100)(\theta$ $=0.5$ ), the dephasing will change the nature of the energy relaxation from being coherent at low temperature to incoherent at "high" (say, $T=150 \mathrm{~K}$ ) temperatures. It would be interesting to perform a detailed theoretical and experimental study of this system.

There is a second very important class of problems where all the phonon modes in the overlayer are involved. For example, experiments have been performed on adsorbates on metal surfaces where an ultrashort laser pulse first excites the electron "gas" at the metal surface, and where "hot" electron plasma interacts with the adsorbed molecules and excites low-frequency adsorbate vibrations. In this case the correct picture is probably to visualize the excitation to occur in a stochastic, incoherent way, and one therefore expects all the q-phonon modes to be equally excited. This problem has usually been treated as a local "heating" problem as follows: ${ }^{22,23}$ if both electrons and phonons contribute to the damping of the adsorbate vibrational mode so that the effective damping $\eta=\eta_{\mathrm{eh}}+\eta_{\mathrm{ph}}$, and if the temperature of the phonon system $\left(T_{\mathrm{ph}}\right)$ and of the electron system $\left(T_{\mathrm{e}}\right)$ are different (as is typically the case after exposing the system to a laser pulse), then it can be shown that within a local picture of (incoherent) adsorbate-substrate energy transfer the average occupation $\bar{n}$ of a low-frequency adsorbate mode will obey the equation

$$
\frac{d \bar{n}}{d t}=\eta_{\mathrm{eh}}\left[n_{e}(t)-\bar{n}\right]+\eta_{\mathrm{ph}}\left[n_{\mathrm{ph}}(t)-\bar{n}\right],
$$

where $\quad n_{\mathrm{e}}=\left[\exp \left(\hbar \omega_{0} / k_{B} T_{\mathrm{e}}\right)-1\right]^{-1} \quad$ and $\quad n_{\mathrm{ph}}$ $=\left[\exp \left(\hbar \omega_{0} / k_{B} T_{\mathrm{ph}}\right)-1\right]^{-1}$ are the Bose-Einstein factors for the electron and phonon systems, respectively, which depend on time via the electron and phonon temperatures $T_{\mathrm{e}}(t)$ and $T_{\mathrm{ph}}(t)$, respectively. In Eq. (54') one must interpret $\eta_{\mathrm{ph}}$ as the average damping, which is approximately given by the damping of an isolated adsorbate. This point was overlooked in a recent paper by Lewis et al., ${ }^{10}$ where the phononic damping of the parallel frustrated translational mode for $\mathrm{CO}$ on $\mathrm{Cu}(100)[c(2 \times 2) \mathrm{CO}$ overlayer], as deduced from ex- 
perimental data of the type described above, was compared with the damping $\eta_{\mathbf{q}}$ evaluated for $\mathbf{q}=\mathbf{0}$.

More generally, whenever the excitation of the lowfrequency modes occurs incoherently, one expects the relevant damping to be well approximated by the average (over the first Brillouin zone) which, as shown above, is (approximately) identical to the damping of a single (isolated) adsorbate. This should also be the relevant damping when considering problems such as the solid-fluid heat transfer ${ }^{2}$ or the dephasing of high-frequency modes by low-frequency modes (e.g., dephasing of the $\mathrm{C}-\mathrm{O}$ stretch vibration by thermal excitation of the low-frequency frustrated translational modes). We note that the original theory ${ }^{6}$ was developed with exactly these types of applications in mind.

\section{INCOMMENSURATE SLIDING LAYER}

It has recently been shown ${ }^{27}$ that incommensurate layers of physisorbed atoms and molecules can slide on smooth metal surfaces, even when the external driving force (per adsorbate) is extremely small (of order $10^{-8} \mathrm{eV} / \AA$ ) . This implies that there is no (or negligible) pinning by the corrugated substrate potential. As shown by Aubry, ${ }^{24}$ this is the case only if amplitude $2 U_{0}$ of the lateral corrugation of the adsorbate-substrate interaction potential is smaller than an effective elastic energy $\mathcal{E}$ associated with the incommensurate solid layer (see, e.g., Ref. 2). Thus, there are two distinct types of incommensurate configurations, a pinned state for "small" $\mathcal{E} / 2 U_{0}$ and a unpinned state for "large" $\mathcal{E} / 2 U_{0}$, and the transition between them (as a function of $\mathcal{E} / 2 U_{0}$ ) is associated with a novel type of continuous phase transition which has been termed "the breaking of analyticity." ${ }^{24}$ In contrast to the periodic substrate potential, one can show that randomly distributed point defects always gives rise to pinning, ${ }^{2}$ but we will assume a perfect surface in the following discussion.

Assume that no pinning occurs. In this case the friction force which acts on the sliding layer from the substrate is proportional to the sliding velocity $\mathbf{v}$ (for small $v$ ); i.e., the friction force per adsorbate has the form $-m \bar{\eta} \mathbf{v}$. There are three fundamental contributions to $\bar{\eta}$ (see Ref. 25), namely, from the direct transfer of kinetic energy from the center-ofmass motion of the layer to the substrate via excitation of (a) low-energy electron hole pairs ${ }^{26}$ and (b) phonons, and (c) from the excitation of phonons in the sliding film which later (perhaps after thermalization of the energy in the overlayer) is transferred to the substrate via process (a) or (b); see Fig. 11. It has been shown that processes (a) and (c) are very important, while it has been argued that process (b) is unimportant, mainly because for an incommensurate layer one expects destructive interference of the elastic waves emitted into the substrate. The aim of this section is to show that this is indeed the case.

Let us assume that the nearest-neighbor adsorbateadsorbate interaction energy is much stronger than the lateral corrugation of the substrate potential and that the adsorbate layer forms an incommensurate structure which, for simplicity, we take to be a square structure (lattice constant $b$ ) (the basic result found below does not depend on this assumption or on any of the other assumptions made below). Assume that the surface unit cell of the substrate is a square (lattice

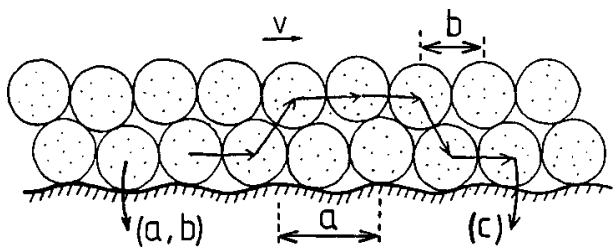

FIG. 11. An adsorbate bilayer sliding on a surface. Two processes contribute to the friction force acting on the bilayer. In (a),(b) a direct process occurs where the translational kinetic energy of the bilayer is directly transferred to the substrate via excitation of (a) low-energy electron-hole pairs or (b) phonons. Process (c) is an indirect process where the corrugated substrate potential first excites vibrations in the bilayer, which are damped via processes (a) and (b).

constant $a$, where $a / b$ is an irrational number), and introduce a coordinate system with the $x$ axis along one side of the surface unit cell. We assume that the overlayer slides in the $x$ direction, and that an atom in the sliding layer experiences a corrugated substrate potential which we assume is of the form $\left(k_{0}=2 \pi / a\right)$ (see Fig. 11)

$$
U(\mathbf{x})=U_{0}\left[\cos \left(k_{0} x\right)+\cos \left(k_{0} y\right)\right],
$$

so that the adsorbate-substrate force in the $x$ direction will be $F=k_{0} U_{0} \sin \left(k_{0} x\right)$. Let us write the position vector of an adsorbate in the sliding layer as

$$
\mathbf{R}=\left(n_{x} b+v t, n_{y} b\right)=\mathbf{n} b+\mathbf{v} t,
$$

where $\mathbf{n}=\left(n_{x}, n_{y}\right)\left(n_{x}\right.$ and $n_{y}$ are integers $)$ and $\mathbf{v}=(v, 0)$. The surface stress $\sigma_{13}$ the sliding layer will exert on the substrate is given by

$$
\begin{aligned}
\sigma_{13}= & k_{0} U_{0} \sum_{\mathbf{n}} \sin \left[k_{0}\left(n_{x} b+v t\right)\right] \delta\left(x-n_{x} b-v t\right) \delta\left(y-n_{y} b\right) \\
= & k_{0} U_{0} \operatorname{Im} \sum_{\mathbf{n}} e^{i k_{0}\left(n_{x} b+v t\right)} \delta\left(x-n_{x} b-v t\right) \delta\left(y-n_{y} b\right) \\
= & \frac{k_{0} U_{0}}{4 \pi^{2}} \operatorname{Im} \int d^{2} k \sum_{\mathbf{n}} \\
& \times e^{i k_{0}\left(n_{x} b+v t\right)} e^{i\left[k_{x}\left(x-n_{x} b-v t\right)+k_{y}\left(y-n_{y} b\right)\right]} \\
\sim & \operatorname{Im} \int d^{2} k e^{i(\mathbf{k} \cdot \mathbf{x}-\omega t)} \sum_{\mathbf{G}} \delta\left(k_{x}-k_{0}-G_{x}\right) \delta\left(k_{y}-G_{y}\right),
\end{aligned}
$$

where $\omega=\left(k_{x}-k_{0}\right) v$. It is clear that this surface stress will generate a displacement field in the bulk of the solid which is a sum of a longitudinal and a transverse part of the form

$$
\begin{aligned}
\mathbf{u} \sim \sum_{\mathbf{G}} \int d^{2} k \mathbf{A}(\mathbf{k}) e^{i(\mathbf{k} \cdot \mathbf{x}+p z-\omega t)} \\
\quad \times \delta\left(k_{x}-k_{0}-G_{x}\right) \delta\left(k_{y}-G_{y}\right),
\end{aligned}
$$

where

$$
p=\left(\frac{\omega^{2}}{c^{2}}-k^{2}\right)^{1 / 2}
$$


and $c$ denotes the transverse or longitudinal sound velocity. Thus,

$$
\mathbf{u} \sim \sum_{\mathbf{G}} \mathbf{A}\left(k_{0}+G_{x}, G_{y}\right) e^{i\left(k_{0}+G_{x}\right) x+i G_{y} y-|p| z-i \omega t},
$$

where $\omega=G_{x} v$, and where we have used that, for $v \ll c$, in general $\left|k_{0}+G_{x}\right|>\left|G_{x}\right| v / c$, so that

$$
\begin{aligned}
p & =\left(\frac{\omega^{2}}{c^{2}}-\left(k_{0}+G_{x}\right)^{2}-G_{y}^{2}\right)^{1 / 2} \\
& =i\left(\left(k_{0}+G_{x}\right)^{2}+G_{y}^{2}-\frac{\omega^{2}}{c^{2}}\right)^{1 / 2}=i|p| .
\end{aligned}
$$

Thus, the displacement field decays exponentially into the substrate; i.e., no energy is radiated into the substrate. Physically, this result is caused by destructive interference of the elastic waves excited by the sliding film. Note, however, that the substrate atoms perform oscillations in the surface region of the solid, and if one were to introduce a damping term in the equation of motion for the elastic media, then energy would be transferred from the sliding layer to the substrate. However, in most cases the damping of the elastic waves is very weak, and can be neglected. If the condition $\left|k_{0}+G_{x}\right|$ $<\left|G_{x}\right| v / c$ is satisfied for at least one $G_{x}$, then energy will be radiated into the elastic half-space from the sliding layer, and a friction force will act on the sliding layer. However, in the measurements by Dayo, Alnasrallah, and $\mathrm{Krim}^{27} v$ $\approx 1 \mathrm{~cm} / \mathrm{s}$, and since typically $c \approx 1000 \mathrm{~m} / \mathrm{s}$, it follows that $v / c \approx 10^{-5}$. It is therefore extremely unlikely that, for an incommensurate sliding layer, the condition $\left|k_{0}+G_{x}\right|$ $<\left|G_{x}\right| v / c$ will be satisfied for any $G_{x}$. On the other hand, for a commensurate sliding layer $k_{0}=G_{x}$ for one $G_{x}$, and the static and kinetic friction would be nonvanishing. This limiting case was studied in Sec. IV.

Finally, we note that process (c) above is a much more important friction channel than process (b). Nevertheless, for a perfect system (no lattice imperfections or lattice anharmonicity) channel (c) will not give rise to any energy dissipation either. Thus, during sliding the atoms in the overlayer film will perform oscillations (around the lattice sites in the moving reference frame) induced by the interaction with the substrate, but in the absence of phonon-damping processes no net energy transfer away from the center-of-mass motion is possible (the phonons excited in the sliding film are virtual). However, because of lattice imperfections and anharmonicity in the sliding layer, and (for metals) because of the electronic damping channel (a) (which damps the lattice vibrations in the overlayer), the "virtual" phonons can become "real," in which case the coupling channel (c) will give a nonvanishing contribution to the sliding friction. This contribution is much larger than that from process (b), mainly because the overlayer (usually $\mathrm{Xe}, \mathrm{Kr}$, or $\mathrm{N}_{2}$ ) films are elastically much softer than the substrate (see Ref. 25). Indeed, computer simulations ${ }^{25}$ indicate that process (c) dominates the sliding friction for uncompressed incommensurate (IC) adsorbate layers, while the electronic friction channel (a) may dominate for compressed IC monolayers. ${ }^{25}$ This conclusion is supported by recent measurements on superconducting lead. ${ }^{13}$

\section{ROLE OF THE ELECTRONIC FRICTION}

In a recent paper Dayo, Alnasrallah and $\mathrm{Krim}^{13}$ report on measurements of the friction force on a thin [1.6 monolayer (ML)] film of nitrogen molecules adsorbed on a lead film. They observed an abrupt drop of the friction force when cooling the lead film below its superconducting temperature $T_{c} \approx 5 \mathrm{~K}$. This indicates clearly the importance of the electronic contribution to friction.

In most experimental investigations on the damping of low-frequency adsorbate vibrations reported so far, the phononic damping dominates (see Sec. VI). For example, this seems to be the case for noble-gas adlayers on $\operatorname{silver}^{28}$ as well as for alkanes adsorbed on a lead surface. ${ }^{29}$ Recently, however, it has been demonstrated that for a special system-saturated hydrocarbons adsorbed on a clean $\mathrm{Ru}(0001)$ surface-damping via electron-hole pair creation dominates over phononic damping. ${ }^{5}$ Furthermore, as discussed above, the phononic friction is strongly suppressed for incommensurate adsorbate layers.

The experimental observation ${ }^{13}$ that the sliding friction for incommensurate $\mathrm{N}_{2}$ overlayers on lead varies upon transition of the substrate to the superconducting state confirms predictions about the importance of electronic friction, ${ }^{30,31}$ but the abruptness of the change in the friction with temperature is entirely unexpected. Since the transition to the superconducting state is a continuous phase transition, one would expect a continuous change in the electronic friction. ${ }^{32}$ The observed results urgently call for a theoretical interpretation. Such an analysis, however, requires precise knowledge of the nitrogen-substrate interface.

According to the experimental description provided by Dayo, Alnasrallah, and Krim the lead substrates were prepared by evaporation ex situ before installing them in the crystal microbalance apparatus after exposing to air for about 10 min. No surface-analytical tools were applied and the exact topography and cleanliness of the substrate surface are thus unknown. Here, we demonstrate that lead samples prepared according to their prescription are covered by a lead oxide/hydrocarbon adlayer of about $15 \AA$ thickness. This numeric value is obtained by quantitatively analyzing the $\mathrm{x}$-ray photoelectron spectroscopy (XPS) data.

The present experiment was performed in a Leybold MAX200 based UHV surface analysis apparatus. The instrument is equipped with a load-lock system which allows the introduction of samples from the ambient into the UHV analysis chamber (base pressure $10^{-10}$ mbar). The polycrystalline $\mathrm{Pb}$ sample (99.999\%) which had been mechanically polished was cleaned in UHV by $\mathrm{Ar}^{+}$sputtering until no contaminations were visible in the XP spectra [see Fig. 12(a) ]. As shown in Fig. 12(b) the XP spectra reveal significant changes after exposing the sample to air for $10 \mathrm{~min}$ and transferring it back into the analytic chamber: the $\mathrm{Pb} 4 f_{7 / 2}$ line is found to be attenuated to $60 \%$ of its original value and additionally $\mathrm{O} 1 s$ and $\mathrm{C} 1 s$ signals are observed. The oxygen line indicates the formation of $\mathrm{PbO}$, while the $\mathrm{C} 1 s$ signal revealed the presence of a weakly adsorbed hydrocarbon overlayer. The latter phenomenon is routinely observed when exposing metal substrates (e.g., Au) to air and is due to the adsorption of saturated and unsaturated long-chain hydrocarbons. ${ }^{33}$ Using a mean free electron path of about 


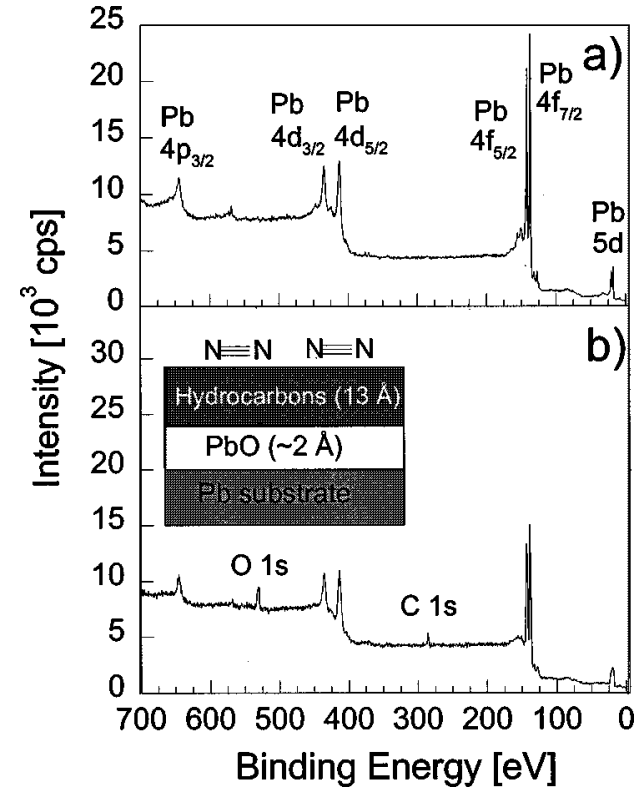

FIG. 12. X-ray photoelectron spectra of (a) a clean $\mathrm{Pb}$ sample and (b) the same sample after exposure to air for $10 \mathrm{~min}$. The inset shows a model of the interface deduced from the relative ratio of $\mathrm{C}$ $1 s$ to $\mathrm{O} 1 s$ signals and the attenuation of the $\mathrm{Pb} 4 f_{7 / 2}$ signal.

$25 \AA$, a value commonly used for organic overlayers, ${ }^{33}$ the attenuation of the $\mathrm{Pb} 4 f_{7 / 2}$ signal corresponds to a total thickness for the $\mathrm{PbO} /$ hydrocarbon adlayer of $15 \AA$. Taking into account the atomic sensitivity factors of the XPS lines, ${ }^{34}$ we estimate from the relative ratio of the $\mathrm{C} 1 s$ to $\mathrm{O} 1 s$ signal a thickness ratio of the $\mathrm{PbO}$ to the hydrocarbon adlayer of 1:6. The corresponding interface structure is shown schematically in the inset of Fig. 12.

Thus we conclude that the $\mathrm{N}_{2}$-adsorption experiments reported by Dayo, Alnasrallah, and Krim were conducted on a lead/lead-oxide/hydrocarbon composite system, which needs to be considered when attempting to theoretically interpret their data. Recently, Persson and Tosatti ${ }^{32}$ pointed out that the present theory of friction fails to explain their observation and thus the abrupt jump in the friction remains puzzling. They suggested that the presence of structural defects on the lead surface may strongly influence the experimental results. Here we demonstrate that a more important effect has to be considered, namely, a significant contamination of the surfaces studied with hydrocarbons. Therefore, in order to study in detail the influence of superconductivity on friction, it would be highly desirable to record comparable data for a well-defined $\mathrm{N}_{2}$ layer (or another well-defined molecular layer) adsorbed on a clean structually well-defined lead substrate, since only in this case the route for a theoretical analysis is straightforward.

\section{SUMMARY AND CONCLUSIONS}

We have presented a study of the damping of lowfrequency adsorbate vibrational modes. When the resonance frequency $\omega_{0}$ of an adsorbate mode is below the highest phonon frequency $\omega_{c}$ of the substrate, it forms a resonance state in the bulk phonon continuum with a contribution to the width coming from the decay by excitation of one bulk (or surface) phonon. If $\omega_{0}$ falls in the linear part of the disper- sion relation of the bulk phonons (which usually is the case if $\left.\omega_{0}<0.3 \omega_{c}\right)$, then the phononic damping can be calculated accurately using the elastic continuum model. For an isolated adsorbate such a calculation was performed in Ref. 6 while ordered structures were considered in Refs. 7-10. In this paper we have extended the earlier studies by considering (a) the influence of the size of the adsorbates on the decay rate, (b) the role of additional adsorbate-adsorbate interactions, and (c) a discussion of how the phononic friction influences different surface processes. We have found the following.

(a) The size of the adsorbates is important for a "large", adsorbate, where the lateral extent of the molecules in at least one spatial direction is larger than $c_{T} / \omega_{0}$. This is the case for many hydrocarbons (e.g., hydrocarbons used as lubricants), some of which have recently been studied using inelastic helium scattering. The size of the adsorbate is not important (even for very large molecules) for the damping of the $\mathbf{q}=\mathbf{0}$ collective mode of ordered adsorbate structures (with small enough lattice constant).

(b) The damping of low-frequency adsorbate vibrations in ordered adsorbate layers is not influenced by any additional (nonphononic) coupling between the adsorbates. The renormalized resonance frequency which enters in the formula for the damping rate of the $\mathbf{q}=\mathbf{0}$ collective phonon mode has no contribution from the additional lateral interaction. Since the lateral interaction does not influence the resonance frequency of the $\mathbf{q}=\mathbf{0}$ phonon mode, this is the correct resonance frequency to be used in the expression for the adsorbate damping.

(c) Some experiments such as inelastic helium scattering or electron energy loss spectroscopy can, if the resolution is high enough, be used to probe the line width of lowfrequency adsorbate vibrations. If the measurements are performed on ordered layers and resolved in $\mathbf{q}$ space, the comparison with theory is simple and straightforward, at least if the energy relaxation rate is much faster than the (pure) dephasing rate (see above). However, in many applications the collective phonon modes are excited incoherently, e.g., in experiments involving surface heating with ultrashort laser pulses, in the heat transfer between a fluid and a solid, or in the dephasing of high-frequency adsorbate modes by anharmonic coupling to thermally excited low-frequency adsorbate modes, and in these cases the situation is less clear. In most of these latter cases it should, however, be a good approximation to use the phonon damping averaged over the whole adsorbate Brillouin zone, which we have shown to be (approximately) equal to the damping experienced by a single (isolated) adsorbate.

We have presented very simple derivations of the damping of the $\mathbf{q}=\mathbf{0}$ mode of ordered adsorbate layers in terms of a model based on a uniform (in space) oscillating (in time) surface stress which emits longitudinal (for perpendicular adsorbate vibrations) or transverse (for parallel adsorbate vibrations) bulk phonons which propagate perpendicular to the surface into the solid. We have also briefly discussed the damping of adsorbate vibrations in disordered adsorbate layers and the sliding of incommensurate layers; in the latter case, because of destructive interference of the emitted elastic waves, there is no damping derived from phonon excitation in the substrate. 


\section{ACKNOWLEDGMENTS}

B.N.J.P. would like to thank the warm hospitality of ICTP and SISSA during a one month stay where most of this work was performed. He also thanks $\mathrm{BMBF}$ for a grant related to the German-Israeli Project Cooperation “'Novel Tribological Strategies from the Nano-to Meso-Scales.' Work at SISSA (E.T.) is partly sponsored by INFM under PRA LORUS and by MURST under Contract No. COFIN97.
${ }^{1}$ B.N.J. Persson, Chem. Phys. Lett. 149, 278 (1988).

2 See, e.g., B.N.J. Persson, Sliding Friction: Physical Principles and Applications (Springer, Heidelberg, 1998).

${ }^{3}$ B.N.J. Persson, F.M. Hoffmann, and R. Ryberg, Phys. Rev. B 34, 2266 (1986).

${ }^{4}$ B.N.J. Persson, Phys. Rev. B 44, 3277 (1991).

${ }^{5}$ G. Witte, K. Weiss, P. Jakob, J. Braun, K.L. Kostov, and Ch. Wöll, Phys. Rev. Lett. 80, 121 (1998).

${ }^{6}$ B.N.J. Persson and R. Ryberg, Phys. Rev. B 32, 3586 (1985).

${ }^{7}$ B. Hall, D.L. Mills, and J.E. Black, Phys. Rev. B 32, 4932 (1985); B. Hall, D.L. Mills, P. Zeppenfeld, K. Kern, U. Becher, and G. Comsa, ibid. 40, 6326 (1989).

${ }^{8}$ L.W. Bruch and F.Y. Hansen, Phys. Rev. B 55, 1782 (1997).

${ }^{9}$ S.P. Lewis and A.M. Rappe, Phys. Rev. Lett. 77, 5241 (1996).

${ }^{10}$ S.P. Lewis, M.V. Pykhtin, E.J. Mele, and A.M. Rappe, J. Chem. Phys. 108, 1157 (1998).

${ }^{11}$ J. Sokoloff, J. Phys. C 10, 9991 (1998).

${ }^{12}$ T.A. Germer, J.C. Stephenson, E.J. Heilweil, and R.R. Cavanagh, J. Chem. Phys. 101, 1704 (1994).

${ }^{13}$ A. Dayo, W. Alnasrallah, and J. Krim, Phys. Rev. Lett. 80, 1690 (1998).

14 R.E. Walkup, D.M. Newns, and Ph. Avouris, in Atomic and Nanometer-Scale Modification of Materials: Fundamentals and Applications, edited by Ph. Avouris (Kluwer, Dordrecht, 1993), p. 100.

${ }^{15}$ D. Fuhrmann and Ch. Wöll, New Journal of Physics 1, 1.1 (1998) (http://www.njp.org/).

${ }^{16}$ J. Ellis, J.P. Toennies, and G. Witte, J. Chem. Phys. 102, 5059 (1995).

${ }^{17}$ A. Graham, F. Hofmann, and J.P. Toennies, J. Chem. Phys. 104, 5311 (1996).
${ }^{18}$ D. Fuhrmann and Ch. Wöll, Surf. Sci. 377-379, 544 (1997).

${ }^{19}$ P. Zeppenfeld, U. Becher, K. Kern, R. David, and G. Comsa, Phys. Rev. B 41, 8549 (1990).

${ }^{20}$ J. Weckesser, K. Weiss, and Ch. Wöll, THEOCHEM 458, 143 (1999).

21 D. Fuhrmann, R. Gerlach, H.-G. Rubahn, and Ch. Wöll, Surf. Sci. (to be published).

${ }^{22}$ B.N.J. Persson and J.W. Gadzuk, Surf. Sci. 410, L779 (1998).

${ }^{23}$ F. Budde, T.F. Heinz, A. Kalamarides, M.M.T. Loy, and J.A. Misewich, Surf. Sci. 283, 143 (1993).

24 S. Aubry, in Solitons in Condensed Matter, edited A.R. Bishop and T. Schneider (Springer, Berlin, 1978), p. 264; W. Selke, in Phase Transitions and Critical Phenomena, edited by C. Domb and J.L. Lebowitz (Academic Press, London, 1992), Vol. 15, p. 1.

${ }^{25}$ B.N.J. Persson and A. Nitzan, Surf. Sci. 367, 261 (1996).

${ }^{26}$ B.N.J. Persson and A.I. Volokitin, J. Phys. C 9, 2869 (1997).

${ }^{27}$ A. Dayo, W. Alnasrallah, and J. Krim, Phys. Rev. Lett. 80, 1690 (1998).

${ }^{28}$ M.S. Tomassone, J.B. Sokoloff, A. Widom, and J. Krim, Phys. Rev. Lett. 79, 4798 (1997).

${ }^{29}$ D. Fuhrmann and Ch. Wöll, Surf. Sci. 368, 20 (1996).

${ }^{30}$ B.N.J. Persson and A.I. Volokitin, J. Chem. Phys. 103, 8679 (1995).

${ }^{31}$ S. Tomassone and A. Widom, Phys. Rev. B 56, 4938 (1997).

${ }^{32}$ B.N.J. Persson and E. Tosatti, Surf. Sci. Lett. 411, L855 (1998).

${ }^{33}$ O. Dannenberger, K. Weiss, H.-J. Himmel, B. Jäger, M. Buck, and Ch. Wöll, Thin Solid Films 307, 183 (1997).

${ }^{34}$ Handbook of X-Ray Photoelectron Spectroscopy, edited by G.E. Muilenberg (Perkin-Elmer Corporation, Eden Prairie, 1978). 